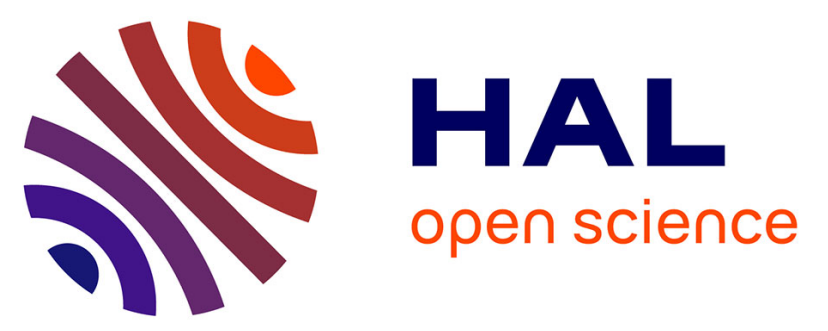

\title{
SURF: A Distributed Channel Selection Strategy for Data Dissemination in Multi-Hop Cognitive Radio Networks
}

Mubashir Husain Rehmani, Aline Carneiro Viana, Hicham Khalife, Serge Fdida

\section{To cite this version:}

Mubashir Husain Rehmani, Aline Carneiro Viana, Hicham Khalife, Serge Fdida. SURF: A Distributed Channel Selection Strategy for Data Dissemination in Multi-Hop Cognitive Radio Networks. [Research Report] RR-7628, INRIA. 2011. inria-00596224v3

\section{HAL Id: inria-00596224 https://hal.inria.fr/inria-00596224v3}

Submitted on 31 Jul 2011

HAL is a multi-disciplinary open access archive for the deposit and dissemination of scientific research documents, whether they are published or not. The documents may come from teaching and research institutions in France or abroad, or from public or private research centers.
L'archive ouverte pluridisciplinaire HAL, est destinée au dépôt et à la diffusion de documents scientifiques de niveau recherche, publiés ou non, émanant des établissements d'enseignement et de recherche français ou étrangers, des laboratoires publics ou privés. 
INSTITUT NATIONAL DE RECHERCHE EN INFORMATIQUE ET EN AUTOMATIQUE

\section{SURF: A Distributed Channel Selection Strategy for Data Dissemination in Multi-Hop Cognitive Radio Networks}

Mubashir Husain Rehmani — Aline Carneiro Viana — Hicham Khalife — Serge Fdida

$\mathbf{N}^{\circ} 7628$

July 2011

Thème COM 



\title{
SURF: A Distributed Channel Selection Strategy for Data Dissemination in Multi-Hop Cognitive Radio Networks
}

\author{
Mubashir Husain Rehmani* , Aline Carneiro Viana ${ }^{\dagger}$, Hicham Khalife $^{\ddagger}$ \\ , Serge Fdida ${ }^{\S}$ \\ Thème COM — Systèmes communicants \\ Équipes-Projets Asap \\ Rapport de recherche $\mathrm{n}^{\circ} 7628$ - July $2011-48$ pages
}

\begin{abstract}
In this paper, we propose an intelligent and distributed channel selection strategy for efficient data dissemination in multi-hop cognitive radio network. Our strategy, SURF, classifies the available channels and uses them efficiently to increase data dissemination reliability in multi-hop cognitive radio networks. The classification is done on the basis of primary radio unoccupancy and of the number of cognitive radio neighbors using the channels. Through extensive NS-2 simulations, we study the performance of SURF compared to three related approaches. Simulation results confirm that our approach is effective in selecting the best channels for efficient communication (in terms of less primary radio interference) and for highest dissemination reachability in multi-hop cognitive radio networks.
\end{abstract}

Key-words: multi-hop cognitive radio networks, dynamic channel selection, data dissemination.

\footnotetext{
* LIP6/Universite Pierre et Marie Curie (UPMC) - Sorbonne Universités, Paris, France

$\dagger$ INRIA, France and TU-Berlin, Germany

¥ LaBRI/ENSEIRB, Université de Bordeaux, France

$\S$ LIP6/Universite Pierre et Marie Curie (UPMC) - Sorbonne Universités, Paris, France
}

\author{
Centre de recherche INRIA Saclay - Île-de-France \\ Parc Orsay Université \\ 4, rue Jacques Monod, 91893 ORSAY Cedex \\ Téléphone : +33172925900
}




\title{
SURF: Une stratégie distribuée de sélection des canaux pour la diffusion de données dans les réseaux radio cognitifs multi-sauts
}

\begin{abstract}
Résumé :
Nous présentons dans cet article une nouvelle stratégie de sélection de fréquences pour la dissémination fiable des données dans le réseau radio cognitifs multi-sauts. En explorant dynamiquement les ressources résiduelles sur les fréquences des primaires et en contrôlant le nombre de CR sur une fréquence particuliére, notre stratégie SURF permet d'augmenter la fiabilité de diffusion des données dans les réseaux radio cognitif multi-sauts. Grâce à une large étude basées sur des simulations sur NS-2, nous étudions les performances de SURF par rapport à trois autres approches liées. Les résultats de simulation confirment que notre stratégie permet la sélection des meilleures fréquences adaptées à une dissémination fiable et efficace des données dans un réseau radio cognitifs multi-sauts.
\end{abstract}

Mots-clés : Réseaux radio cognitifs multi-sauts, sélection dynamique de fréquence, dissémination de données. 


\section{Introduction}

Data dissemination is commonly defined as the spreading of information to multiple destinations through broadcasting. The main objective is to reach the maximum number of neighbors with every sent packet. In this communication scheme, no routing is required thus neither routing tables nor end-to-end paths are maintained. Among different applications where data dissemination can be useful, we focus in this work on networking scenarios where providers disseminate non-urgent messages in order to limit cost and complexity through the network, such as: services, updates (e.g., new code to re-task a provided service), or any kind of publicity message. However, guaranteeing reliability of data dissemination in wireless networks is a challenging task. Indeed, the characteristics and problems intrinsic to the wireless links add several issues in the shape of message losses, collisions, and broadcast storm problem, just to name a few.

In this paper, we focus on data dissemination in ad hoc cognitive radio networks. Cognitive radio networks are composed of cognitive radio devices. The concept of cognitive radio was introduced in the seminal paper by J. Mitola [1]. The motivation behind cognitive radio was threefold: (1) availability of limited spectrum, (2) fixed spectrum assignment policy, and (3) inefficiency in spectrum usage. Therefore, cognitive radio networks are designed to opportunistically exploit the underutilized spectrum. Moreover, the regulatory bodies, such as, the Federal Communication Commission (FCC) [2] also promoted the idea of using the cognitive radio devices to address the spectrum shortage problem. In this regard, the FCC has designed an interference-free opportunistic spectrum access policy [2]. According to the FCC's policy [2], channels are only allowed to be used by Cognitive Radio (CR) nodes if they are idle i.e., not utilized by the Primary Radio (PR) nodes and CR nodes should avoid causing harmful interference to PR nodes. In fact, PR nodes are the legacy users and they have higher priority to use the licensed band. CR nodes can take advantage of idle channels to disseminate non-urgent and publicity messages with low cost and complexity.

Particularly in the context of Cognitive Radio Wireless Networks (CRN) [3], where channels for transmission are opportunistically selected, reliability is difficult to achieve. This is due to the inherent features of such networks. First, in addition to the already known issues of wireless environments, the diversity in the number of channels that each cognitive node can use adds another challenge by limiting node's accessibility to its neighbors. Second, Cognitive Radio (CR) nodes have to compete with the Primary Radio (PR) nodes for the residual resources on channels and use them opportunistically. Besides, CR nodes should communicate in a way that does not disturb the reception quality of PR nodes by limiting CR-to-PR interference [4].

In multi-hop cognitive radio ad-hoc networks, where coordination between CRs is hard to achieve and no central entity for regulating the access over channels is present, reliable data dissemination is even more complex. In this perspective, the important step in having efficient data dissemination is to know how to select best channels. In fact, channel selection plays a vital role in reliable data dissemination. If CR nodes select the channels randomly, there are very less chances that the neighbor receivers also select the same channel. Consequently, the random selection of channels severely degrades the data dissemination reachability. Furthermore, when CR nodes randomly select the channel for transmission, it may be possible that a PR transmission is going on and subsequently, the CR transmission causes harmful interference to the PR nodes.

A lot of works have been carried out for dynamic channel management in cognitive radio networks $[5,6,7,8,9,10,11,12,13,14,15,16]$. These approaches focus on single- 
hop cognitive radio networks $[5,6,10,14]$ and either requires the presence of any central entity $[11,16]$ or the coordination with primary radio nodes in their channel selection decision $[14,16]$. Moreover, these aforementioned channel selection strategies are not specifically designed for data dissemination, e.g., [5] consider the traffic demands of Access Points, [16] discuss throughput maximization, [11] discuss load balancing, just to name a few.

A more related to our approach is Selective Broadcasting (SB) [17]. Selective broadcasting is proposed for multi-hop cognitive radio networks, in which nodes select a minimum set of channels i.e. Essential Channel Set (ECS) to cover all its geographic neighbors. There are however, several challenges in the practicality of SB. From the communication perspective, simultaneous transmission over an ECS requires more than one transceiver, resulting in bigger and more complex devices, as for military applications [18]. Furthermore, transmissions over a set of channels without considering the PR activity may increase the probability of interference with primary radio nodes. Since, no centralized entity is present to synchronize nodes in their channel selection decision, selecting an inappropriate channel for overhearing from the ECS channel set by the neighboring nodes may lead to packet losses. Therefore, a new channel selection strategy is required which works well with single transceiver, cause less harmful interference to PR nodes and try to maximize the chances that the message is delivered to the neighboring cognitive radio receivers, thus increasing the data dissemination reachability.

Thus, differently from works in the literature, we go a step further here and build up a channel selection strategy, SURF, for data dissemination in multi-hop cognitive radio networks. In SURF, the objective of every cognitive radio node is to select the best channel ensuring a maximum connectivity and consequently, allowing the largest data dissemination reachability in the network. This corresponds to the use of channels having low primary radio nodes (PRs) activities, as well as having higher number of CR neighbors.

In SURF, the classification of channels is done on the basis of primary radio unoccupancy and the number of cognitive radio neighbors using the channels. Another main challenge we deal with in this paper reside in making efficient and reliable channel selection decisions on-the-fly and in recovering from bad channel selection decisions. To deal with this challenge, we introduce the mechanism of recovery from bad channel selection decision. In this mechanism, SURF keeps track of previous wrong channel state estimation and accordingly adapts future channel selection decision. Usually channel selection strategies provide a way to nodes to select channels for transmission. Besides, SURF endues CR nodes to select best channels also for overhearing. This will help to tune both sender and receiver with high probability to the same channel. As a consequence, SURF may have high number of neighbors on the selected channel. In addition to that, SURF protects the PR nodes by considering the PR unoccupancy in channel selection decision, for effective and reliable data dissemination.

We analyze the performance of SURF through extensive NS-2 simulations. We use the Cognitive Radio Cognitive Network (CRCN) patch [19] of Network Simulator NS-2 [20]. The CRCN patch of NS-2 does not support the activity of the PR nodes. Thus, we enhance the CRCN patch of NS-2 to include the PR activity model. We compare SURF with Random (RD), Highest Degree (HD) and Selective Broadcasting (SB) approach [17]. In order to evaluate SURF, we use five metrics: (1) harmful interference ratio, which we choose to characterize the probable interference caused by CR transmissions to PR nodes; (2) average delivery ratio and (3) ratio of accumulative CR receivers, both for evaluating the reliability of data dissemination; (4) ratio of effective 
neighbors and (5) ratio of accumulative effective neighbors, both of them are chosen to characterize the tuning of sender/receiver nodes. We comprehensively analyze SURF by varying node density, number of retries, packet drop reasons, etc. Simulation results confirm that SURF protects the PR nodes during transmission compared to RD, $\mathrm{HD}$ and SB approaches. SURF is also able to achieve the average delivery ratio of $40 \%-50 \%$, compared to $0 \%$ in RD, $1 \%$ for SB and $2 \%$ for HD approaches. The results of effective neighbors and accumulative effective neighbors show that SURF is able to well tune the sending/receiving CR nodes and thus, able to create with high probability, a connected topology. Besides these advantages, the simplicity and decentralized nature of SURF makes it usable in ad-hoc CRNs deployed to convey services, updates, or any kind of publicity message.

The major contributions of this paper are summarized in the following:

- We design SURF, an intelligent and distributed channel selection strategy for data dissemination in multi-hop cognitive radio networks. SURF is also equipped with the mechanism of recovery from bad channel selection decision.

- We enhances the Network Simulator NS-2 to include the PR activity model.

- We validate SURF though different metrics and compare with RD, HD, and SB.

- We provide a detailed literature review on channel selection strategies in cognitive radio networks.

The remainder of this paper is organized as follows: we discuss challenges of data dissemination in Section 2. Then we discuss system model and assumptions in Section 3. We give general overview of SURF in Section 4. Section 5 and 6 deal with detailed description of SURF. Performance analysis is done in section 7. Section 8 discusses the activity pattern impact of primary radio nodes on channel selection strategies. Section 9 discusses related work, and finally, section 10 concludes the paper.

\section{Challenges of Data Dissemination in Cognitive Radio}

\section{Networks}

Data dissemination is a classical and a fundamental function in any kind of network. In wireless networks, the characteristics and problems intrinsic to the wireless links bring several challenges in data dissemination in the shape of message losses, collisions, and broadcast storm problem, just to name a few. However, data dissemination is extremely challenging issue in cognitive radio networks due to its intrinsic properties, such as:

- the availability of multiple-channels i.e., CR nodes have more than one channel in the available channel set. More specifically, the id's of the channels in the available channel set of sender and receiver are same.

- the diversity in the number of available channels i.e., CR have more than one channel in the available channel set. But, the id's of the channels in the available channel set of sender and receivers are different.

- the primary radio activity i.e., channels are occupied by the PR nodes and are only available to $\mathrm{CR}$ nodes for transmission when they are idle. In fact, the 
spatiotemporal utilization of spectrum by PR nodes (i.e. primary radio nodes' activity) adds another dimension of complexity to data dissemination. As a consequence, the number of available channels to CR nodes changes with time and location and this leads to the diversity in the number of available channel set. Because of PR's activity, the usability of the channels by CR nodes becomes uncertain.

Moreover, without any centralized entity, as in the case of multi-hop ad hoc cognitive radio network, data dissemination is even more challenging because CR nodes have to rely on locally inferred information for their channel selection decision. If a channel selection is done in an intelligent way, higher data dissemination reachability can be achieved. Furthermore, the consideration of PR activity during channel selection can enhance the effectiveness of data dissemination reachability and can reduce the harmful interference to PR nodes by CR transmissions.

We mention some key required characteristics of any channel selection strategy for data dissemination in cognitive radio networks:

1. Efficient message delivery: A good channel selection strategy is the one that increases the probability of higher message delivery in multi-hop context.

2. Primary radio constraints: The channel selection strategy should ensure that the transmission on the selected channel does not create harmful interference to primary radio nodes.

3. Autonomous decision by CR nodes: It means that the channel selection strategy should work well without any centralized authority and channel selection decision should be based on locally inferred information.

4. Sender/Receiver tuning: The channel selection strategy should guarantee that the CR transmitter and receiver select the same channel with high probability.

\section{System Model and Assumptions}

In this section, we present the system model considered and the basic assumptions related to our proposal.

\subsection{Network Model}

We consider a Cognitive Radio Ad-Hoc Network [21]. In this type of network setting, we assume that no centralized network entity is available. Instead, we consider a networking environment where network operations (e.g., spectrum sensing, channel selection decision etc) are performed by the CR nodes themselves. The network is composed of a set of Primary Radio (PR) nodes and a set of Cognitive Radio (CR) nodes. Primary radio nodes are the licensed users and they can access their respective licensed bands without any restriction. Indeed, PR nodes have the highest priority to access the channels and should not be interrupted by the CR nodes [4].

In order to be able to communicate in a CRN, CR nodes must create a multi-hop network by using the licensed bands. The use of licensed bands by cognitive radio nodes are however, only possible when the bands are idle, i.e. unoccupied by the PR 
nodes. Note that an idle state describes the temporal availability of a channel. In some cases, it can happen that a CR node starts a transmission at the same time when PR becomes active. Since, we consider that $\mathrm{CR}$ transmissions should not generate harmful interference at PR receivers [22], CRs will cancel their transmissions.

We further assume that CR nodes are equipped with a single transceiver. This transceiver can either receive or transmit on a single channel at a time. The utilization of single transceiver reduces the operational cost of the CR device [23], as well as avoids potential interference between co-located transceivers due to their close proximity [24]. We consider the set of total frequency channels $C$.

\subsection{Spectrum Sensing by Cognitive Radio Nodes}

In cognitive radio ad-hoc networks, cognitive radio nodes are assumed to work in stand alone fashion and make decisions based on locally inferred information. As a consequence, each CR node has to perform spectrum sensing to detect the presence of the PR signal. We assume that the spectrum sensing is periodically performed by every CR node. We further assume that the detection of the PR signal is the responsibility of the spectrum sensing block [25]. In this case, SURF will work on the list of available channels resulted from the spectrum sensing.

\subsection{Primary Radio Activity or Wireless Channel Model}

The performance of cognitive radio network is closely related to the primary radio activity over the channels. Therefore, the estimation of primary radio activity plays a vital role in channel selection decision. We assume that the primary radio activity or wireless channel can be modelled as continuous-time, alternating ON/OFF Markov Renewal Process (MRP) [26, 27, 28] (cf. Section 5 for more details). Note that such an ON/OFF PR activity model captures the time period in which the channel can be utilized by CRs without causing any harmful interference to PR nodes [29].

\subsection{Exchange of Hello Packets}

[30] could be used to help the neighbor discovery process and it uses a Common Control Channel (CCC) mechanism. In this CCC mechanism, CR nodes locally make clusters and the control channel from the ISM band is dynamically allocated within each cluster. The reason behind locally making clusters by CR nodes is due to the global unavailability of control channel. In [30], first the neighbor discovery is performed. The neighbor discovery consists of three phases: (1) each CR determines the set of idle channels, (2) a universal time schedule for channel access is followed, and (3) using this universal time schedule, CRs can discover their neighbors. In the second step, clustering is performed based on the set of idle channels that are common to all cluster members. The control channel from a given cluster is selected from this set. In this manner, the goal of increasing the availability of common idle channel in each cluster is achieved by grouping CRs with similar spectrum opportunities.

We assume the availability of a out-of-band Common Control Channel (CCC) [21] for neighbor discovery. Due to the time variability of PR activity on the licensed band, control channel is selected from the unlicensed ISM band. The reason behind selecting a dedicated spectrum band for CCC is to minimize the CCC disruptions caused by PR 
activity. It is worth noting that the cost of switching between data channels and control channel is non-negligible because of the availability of a single transceiver.

\section{Channel Selection Strategy SURF}

The SURF channel selection strategy is specifically designed for ad-hoc cognitive radio networks. The general goal of SURF is to increase reliability in data dissemination over a multi-hop ad hoc CRN. Note that SURF is a packet-based channel selection scheme for data dissemination and not a routing algorithm. Therefore, neither the routing tables nor the end-to-end paths are maintained by the CR nodes. CR nodes, upon each packet reception, select the best channel, and broadcast the packet.

With SURF, every CR node autonomously classifies available channels based on the observed PR-unoccupancy over these channels. This classification is then refined by identifying the number of CRs over each band. The best channel for transmission is the channel that has the higher PR unoccupancy and a higher number of CR neighbors. Indeed, choosing a channel with few CRs may yields to a disconnected network. Every CR after classifying available channels, switches dynamically to the best one and broadcasts the stored message. Moreover, SURF also tries to learn with previous wrong channel state estimation. This learning process allows better tuning the future estimations and helps CR nodes to recover from their bad channel selection decisions.

Additionally, CRs with no messages to transmit implement the SURF strategy in order to tune to the best channel for data reception. Using the same strategy implemented by the sender allows receivers in close geographic areas to select with high probability the same used-to-send channel for overhearing. This will also increase the number of CR neighbors on the selected channel. This is due to the fact that, intuitively, it is likely that CRs in the sender's vicinity have the same PR unoccupancy, hence channels available to a CR sender is also available to its neighbors with high probability [8]. Therefore, SURF increases the probability of creating a connected topology. Once a packet is received, every CR receiver undergoes again the same procedure to choose the appropriate channel for conveying the message to its neighbor.

Channel's Weight Calculation Formula SURF strategy classifies channels by assigning a weight $P_{w}^{(i)}$ to each observed channel $i$ in the channel set $C$. Thus, every cognitive radio node running SURF, locally computes the $P_{w}^{(i)}$ using the following equation:

$$
\forall i \in C: P_{w}^{(i)}=P R_{u}^{(i)} \times C R_{o}^{(i)}
$$

$P_{w}^{(i)}$ describes the weight of a channel $(i)$ and is calculated based on the PR unoccupancy (i.e. $P R_{u}^{(i)}$ ) and CR occupancy (i.e. $C R_{o}^{(i)}$ ) over channel $i$ (c.f. section 5 and section 6). Then, the channels are ranked according to their weights and the best channel (i.e., the one providing highest $P_{w}^{(i)}$ ) will be used. Note that when the channel has high weight but at time $t$ it is occupied, SURF reacts (i) by not transmitting the packet on the best weighted channel and (ii) by selecting the next best weighted channel for packet transmission/overhearing. Also note that when all the channels are occupied, no message is sent.

The increase of weight is related to the two objectives the SURF strategy needs to satisfy. The major objective of protecting the ongoing PR activity is mapped as a 
function of PR unoccupancy. The higher the probability of PRs being in OFF state, i.e. $P R_{u}^{(i)}$, the higher the weight will be. Thus, SURF gives high importance to not degrading the service of ongoing primary communications. The second objective of increasing connectivity is implemented in the second term of Eq.11. More precisely, the weight increases with the number of $\mathrm{CR}$ neighbors i.e. $C R_{o}^{(i)}$. In the following, we discuss in detail how the primary radio unoccupancy and cognitive radio occupancy could be estimated.

\section{Primary Radio Unoccupancy}

The primary radio activity, i.e. presence or absence of the PR signal, can be modelled as continuous-time, alternating ON/OFF Markov Renewal Process (MRP) [26, 27, 28]. This PR activity model has been used very widely in the literature $[26,27,28,31,32,33$, $34,35]$. The ON/OFF PR activity model approximates the spectrum usage pattern of public safety bands $[34,36]$. The public safety band is designated for commercial and public safety uses [37]. The authors in [38] approximate and validate the PR ON/OFF activity model for the presence of the PR signal in IEEE 802.11b. The ON/OFF PR activity model is also the most famous model for voice [39]. An important feature of this ON/OFF PR activity model is that it captures the time period in which the channel can be utilized by CRs without causing any harmful interference to PR nodes [29]. Fig. 1 illustrates the wireless channel model. The ON i.e. busy state indicates that the channel is currently occupied by the PR node, while the OFF i.e. idle state indicates that the channel is currently unoccupied by PR node.



Figure 1: Wireless channel model: Alternating Markov Renewal Process for PR activity.

The duration of ON and OFF states of channel $i$ are denoted as $T_{O N}^{i}$ and $T_{O F F}^{i}$, respectively. The renewal period of a channel occurs when one consecutive $\mathrm{ON}$ and OFF period is completed. Let $Z_{i}(t)$ denote the renewal period of channel $i$ at time $t$, such that $Z_{i}(t)=T_{O F F}^{i}+T_{O N}^{i}[26,28,29,40]$. 
Both ON and OFF periods are assumed to be independent and identically distributed (i.i.d.). Since each PR user arrival is independent, each transition follows the Poisson arrival process. In [26,40], the authors proved that when each PR arrival follows the Poisson arrival process, the length of ON and OFF periods are exponentially distributed. In this paper, we use the formulation of $[26,28,29,40]$ that the channels ON and OFF periods are both exponentially distributed with p.d.f. $f_{X}(t)=\lambda_{X} \times e^{-\lambda_{X} t}$ for ON state and $f_{Y}(t)=\lambda_{Y} \times e^{-\lambda_{Y} t}$ for OFF state.

The duration of time in which channel $i$ is in ON state i.e. channel utilization $u^{i}$ is given as [29] :

$$
u^{i}=\frac{E\left[T_{O N}^{i}\right]}{E\left[T_{O N}^{i}\right]+E\left[T_{O F F}^{i}\right]}=\frac{\lambda_{Y}}{\lambda_{X}+\lambda_{Y}}
$$

where $E\left[T_{O N}^{i}\right]=\frac{1}{\lambda_{X}}$ and $E\left[T_{O F F}^{i}\right]=\frac{1}{\lambda_{Y}} \cdot \lambda_{X}$ and $\lambda_{Y}$ are the rate parameter for exponential distribution. $E\left[T_{O N}^{i}\right]$ and $E\left[T_{O N}^{i}\right]$ is the mean of exponential distribution. Let $P_{O N}(t)$ be the probability of channel $i$ in ON state at time $t$ and $P_{O F F}(t)$ be the probability of channel $i$ in OFF state at time $t$. The probabilities $P_{O N}(t)$ and $P_{O F F}(t)$ can be calculated as:

$$
\begin{aligned}
& P_{O N}(t)=\frac{\lambda_{Y}}{\lambda_{X}+\lambda_{Y}}-\frac{\lambda_{Y}}{\lambda_{X}+\lambda_{Y}} e^{-\left(\lambda_{X}+\lambda_{Y}\right) t} \\
& P_{O F F}(t)=\frac{\lambda_{X}}{\lambda_{X}+\lambda_{Y}}+\frac{\lambda_{Y}}{\lambda_{X}+\lambda_{Y}} e^{-\left(\lambda_{X}+\lambda_{Y}\right) t}
\end{aligned}
$$

Thus, by adding Eq.3 and Eq.13, we get

$$
P_{O N}(t)+P_{O F F}(t)=1
$$

Since our goal is to select the channel that will be unoccupied at time $t$, from hereafter we will only consider $P_{O F F}(t)$. Each CR node locally computes these probabilities. The values of $\lambda_{X}$ and $\lambda_{Y}$ can be easily measured by CR nodes by collecting the historical samples of channel state transitions, as in [29]. In this paper, we are using the values measured by authors in [29] (cf. Table 2).

The best channel at time $t$ is the one that has very high probability of being in OFF state. It may be possible that the probabilistically estimated next channel state mismatch with the current state of the channel, referred hereafter as wrong channel state estimation. This further leads to bad channel selection decision and cause harmful interference to PR nodes. Note that CR nodes keep the history of estimated and measured states of the channel. Next, we detail how the learning of previous wrong estimation can help to tune future estimations.

\subsection{Recovery from Bad Channel Selection Decisions}

Another challenge we deal with in this paper reside in making efficient and reliable channel selection decisions on-the-fly and in recovering from bad channel selection decisions. Clearly, keeping track of wrong channel state estimations can help CR nodes to recover from their bad channel selection decisions, which ultimately enhance the reliability and the performance. Due to the memoryless property of the markov exponential model, there is a large degree of randomness and this result in imperfect prediction of channel state [34]. To deal with this memoryless property of the markov exponential 
model, CR nodes always keep calculating the next state of the channel, $P_{O F F}(t)$, with Equation 13. In parallel, CR nodes calculates $P_{O F F}^{*}(t)$ which considers the current state of the channel and wrong channel state estimations.

To achieve this goal, nodes maintain the history of estimated channel states and the observed current state of the channels. CR nodes then compute which estimations were wrong and keep them in history. This history is then used to calculate the probabilities $P_{U M}$ and $P_{S M} . P_{U M}$ is defined as the probability that the estimated channel state mis-matches with the actual channel state. Each CR node uses $P_{U M}$, while calculating the next channel state (cf. Fig. 2). Conversely, the probability of successfully matched state $P_{S M}$ is defined as the probability that the estimated channel state matches with the current channel state. More precisely, the accuracy of the recovery mechanism of SURF depends upon the estimated state of the channel (cf. probability value given by Eq. (13)) and the measured current state of the channel. Table. 5 provides the possible combinations between the values of estimated state and current state of the channel.

The probability $P_{S M}$ is expressed as:

$$
P_{S M}^{(i)}=\frac{x_{t}}{N}
$$

where $x_{t}$ is the number of times the estimated channel state matches with the actual channel state, and $N$ is the total number of times the estimation occurs, and

The probability $P_{U M}$ is expressed as:

$$
P_{U M}^{(i)}=\frac{x_{n t}}{N},
$$

where $x_{n t}$ is the number of times the estimated channel state does not match with the actual channel state i.e. how often the channel states estimation was erroneous, and $N$ is the total number of times the estimation occurs. In fact, the $P_{U M}$ measures two different types of channel states cases (cf. Table 5). The first one is the case when estimated channel state is OFF and the measured channel state is ON and the second one is the case when the estimated channel state is ON the measured channel state is OFF. Thus, we further decomposed $P_{U M}$ into $P_{M D}$ and $P_{F A}$ as:

$$
P_{U M}^{(i)}=\frac{x_{n t}}{N}=P_{M D}{ }^{(i)}+P_{F A}^{(i)},
$$

where $P_{M D}$ is the Probability of Miss-Detection and occurs when estimated channel state is OFF and the measured channel state is ON. In $P_{M D}$, CR node declares the busy channel as unoccupied. This will lead to harmful interference with PR nodes. While, $P_{F A}$ is the Probability of False-Alarm and occurs when the estimated channel state is $\mathrm{ON}$ and the measured channel state is OFF. In $P_{F A}, \mathrm{CR}$ node declares that the unoccupied channel is busy. This will lead to refrain CR node from transmitting and thus, loose spectrum opportunity. $P_{F A}$ and $P_{M D}$ are measured by every CR node on per channel basis. In fact, $C R$ node estimates the state of the channel and this estimated state is compared with the actual state of the channel. If the estimated state of the channel is $\mathrm{ON}$ and the measured channel state is OFF, CR node increase the $P_{F A}$ counter, else if the estimated state of the channel is OFF and the measured state of the channel is ON, CR node increase the $P_{M D}$ counter. Both the $P_{F A}$ and $P_{M D}$ counters are then divided by the total number of times the estimation occurs. In this manner, each $\mathrm{CR}$ node maintains the history of $P_{F A}$ and $P_{M D}$. 


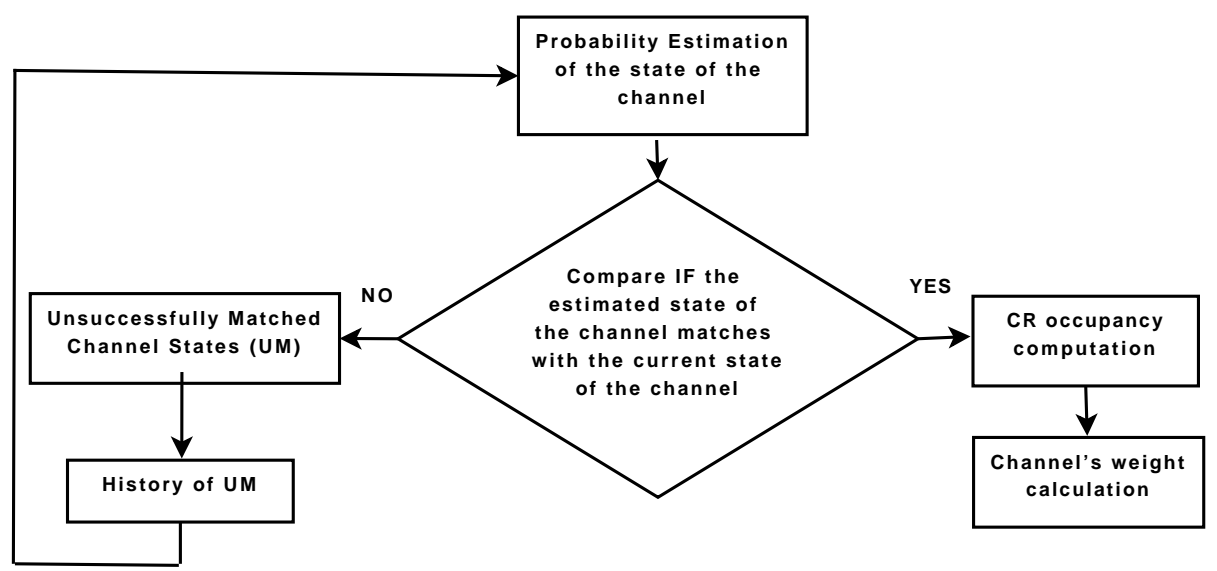

Figure 2: Flow chart showing the corrective measure taken by the CR nodes in the case of detection of unsuccessfully matched channel states i.e. $P_{U M}$.

Table 1: Estimated and Current States of the Channel.

\begin{tabular}{|c|c|c|c|}
\hline \multicolumn{2}{|c|}{ Event } & Estimated State & Current State \\
\hline \multirow{2}{*}{\multicolumn{2}{|c|}{$P_{S M}$}} & ON & ON \\
\hline & & OFF & OFF \\
\hline \multirow[t]{2}{*}{$P_{U M}$} & $P_{M D}$ & OFF & ON \\
\hline & $P_{F A}$ & ON & OFF \\
\hline
\end{tabular}

Consequently, the lower the $P_{U M}(t)$, the more accurate will be the channel state estimation. Putting things together, we estimate $P_{O F F}^{*}(t)$, which considers the probability of unsuccessfully matched state during the channel state estimation, as follows:

$$
P R_{u}^{(i)}=P_{O F F}^{*}(t)^{(i)}=P_{O F F}^{(i)}\left(1-P_{F A}^{(i)}\right)+P_{M D}^{(i)}\left(1-P_{O F F}^{(i)}\right)
$$

In the case of a perfect channel estimation (i.e., $P_{F A}=0$ and $\left.P_{M D}=0\right), P_{O F F}^{*}(t)=P_{O F F}(t)$. In the presence of channel estimation errors, the probability of channel $(i)$ being in OFF state is given by Eq. (12).

\section{Cognitive Radio Occupancy}

CR occupancy reflects the number of CR neighbors using the channel. In fact, a good channel selection strategy is the one that tune CR nodes to the channel that have higher number of CR neighbors. Higher number of CR neighbors provides good level of network connectivity and consequently increase the transmission coverage of CR nodes. The CR occupancy $C R_{o}^{(i)}$ of channel $(i)$ is calculated as:

$$
C R_{o}^{(i)}=C R_{n}^{(i)}
$$


where, $C R_{n}^{(i)}$ is the number of CR neighbors using the channel $(i)$.

In order to calculate the CR occupancy, each CR node discovers their neighbors. Neighbors can be discovered in an efficient way by denominating the Common Control Channel (CCC), which will ensure the availability of common idle channel between CR nodes, and the neighbor discovery mechanism, as in [30]. The authors in [30] assumed that due to the global unavailability of control channel, CR nodes have to locally make clusters that decrease the overhead in neighbor discovery and make the coordination between CR nodes easier. After locally making the clusters by CR nodes, the control channel from the ISM band is dynamically allocated within each cluster. In this manner, the goal of increasing the availability of common idle channel in each cluster is achieved by grouping CRs with similar spectrum opportunities. Consequently, each $\mathrm{CR}$ node is able to calculate the CR occupancy by knowing the number of CR neighbors using the channel. In addition to neighbor discovery mechanism proposed in [30], SURF can jointly work with any other neighbor discovery mechanism, such as $[41,42]$.

\section{Performance Analysis}

In this section, we analyze the performance of SURF through extensive simulations.

\subsection{Implementation Setup}

We use the Cognitive Radio Cognitive Network (CRCN) patch [19] of NS-2 [20]. The CRCN patch has three building blocks that supports cognitive radio functionalities in NS-2 (cf. Fig. 3). These building blocks are the cognitive radio network layer, the cognitive radio mac layer and the cognitive radio physical layer. The cognitive radio network layer is responsible for maintaining the neighbor list. It also makes the channel selection decision on the basis of the information provided by the cognitive radio MAC layer. The cognitive radio MAC layer supports multiple channels and keeps track of PR traffic, collision, interference information and it also maintains the channel list. The cognitive radio physical layer has information like transmission power, SINR/SNR physical model, propagation model etc. The information collected at different layers is shared through the information sharing layer.

This CRCN patch of NS-2 does not support the activity of the PR nodes. Thus, we enhance the CRCN patch of NS-2 to include the PR activity model. Fig. 3 shows the high level design of PR activity model (dotted box) added in NS-2. The PR activity block is responsible for keeping track of PR activities in each spectrum band (spectrum utilization) i.e., sequence of $\mathrm{ON}$ and $\mathrm{OFF}$ periods by $\mathrm{PR}$ nodes over the simulation time. These $\mathrm{ON}$ and $\mathrm{OFF}$ periods can be modelled as continuous-time, alternating ON/OFF Markov Renewal Process (MRP) [26], [28]. The ON (busy) state means the channel is occupied by the PR node. While, the OFF (idle) state means the channel is unoccupied by the PR node. We consider the channels ON and OFF periods are both exponentially distributed, as in [28], [29]. The rate parameter $\lambda_{X}$ and $\lambda_{Y}$ (cf. Table 2) of the exponential distribution is provided as an input in the simulation, which were measured by authors in [29]. Then, according to this rate parameter, channels follow the $\mathrm{ON}$ and $\mathrm{OFF}$ periods.

We consider a simple mac protocol (Maccon.cc), available with the CRCN patch of NS-2. This mac protocol is a multiple-channel, collision and contention-based mac protocol. Note that in the original state, the Maccon.cc mac protocol selects channel 
randomly from the predefined set of channels and the channel selection decision occurs at the mac layer. We now perform channel selection at the network layer. Thus, we modify this mac protocol and provide the capability to the network layer to make the channel selection decision. We further add channel selection strategies RD, HD, SB and SURF to the network layer, which we describe hereafter. Based upon any particular channel selection strategy, the network layer takes the channel selection decision. This channel selection decision is encapsulated in the network layer packet header and it is passed to the mac layer, which then switch to the channel based on the channel selection decision provided by the network layer.

In the Maccon.cc mac protocol, there are two channel states: IDLE and BUSY. These states are dependent on the channel conditions and they have used by the mac protocol to handle the transmission and reception activities of CR nodes. IDLE means that the channel is free to use for transmission by the CR node and BUSY means that the channel is occupied by any undergoing CR transmission. In order to deal with the activities of the PR nodes, we include two more states at mac layer for each channel i.e., PR_OCCUPIED and PR_UNOCCUPIED. The state PR_OCCUPIED means that the channel is occupied by the PR node and PR_UNOCCUPIED means that the channel is unoccupied by the PR node. These two states of the channel will be checked each time by the mac protocol while performing transmission or overhearing.

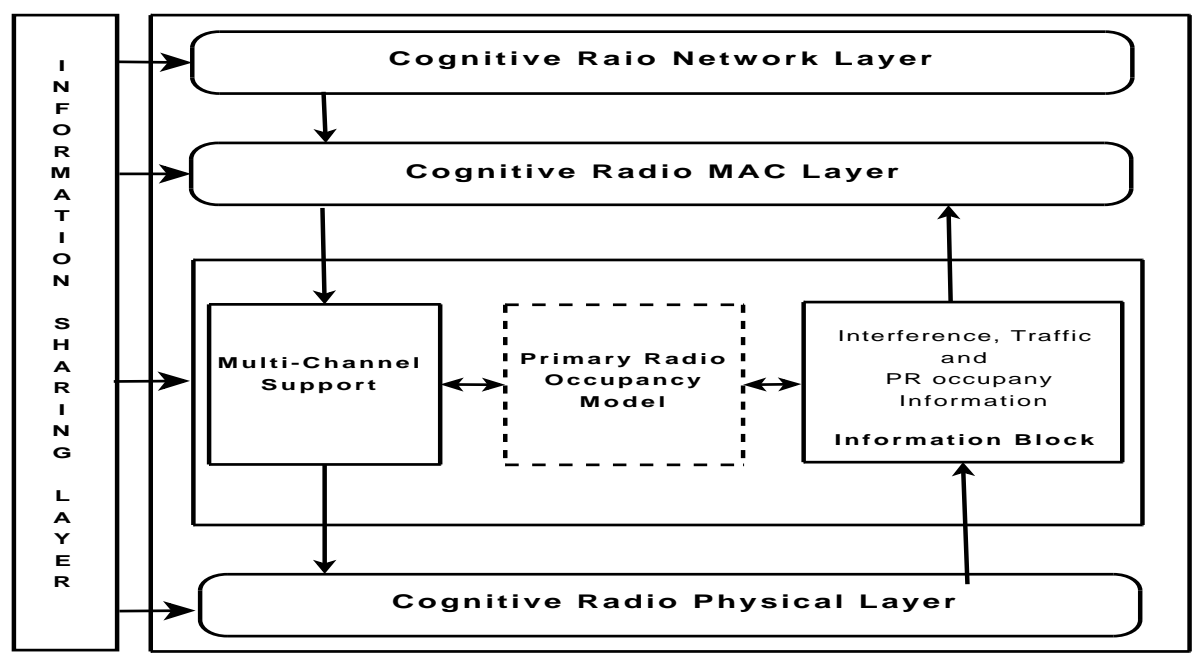

Figure 3: High level design of primary radio activity model in NS-2.

\subsection{Performance Metrics}

We compare SURF with random strategy (RD), highest degree strategy (HD) and selective broadcasting, proposed in [17] with multiple transmissions (SB). We suggested $\mathrm{RD}$ strategy, which is the simplest one and no information is required. In RD, channels are randomly selected to be used by CR nodes for transmission and/or overhearing, without any consideration to the ongoing PR and CR activity over these channels. HD approach only considers CR activities and is inspired by SB approach. In HD, CR nodes select the highest $\mathrm{CR}$ degree channel for transmission and overhearing, without 
any consideration of PR activity. The highest degree channel covers, consequently, the highest number of neighbors in the available list of channels. In SB, each CR node calculates a minimum set of channels, Essential Channel Set (ECS), for transmission that covers all its geographic neighbors, without considering the PR unoccupancy. In $\mathrm{SB}$, a CR node transmits on multiple channels in round-robin fashion present in the ECS list, until all neighbors are covered. Note that in [17] nothing is mentioned about how nodes overhear over the channels. Therefore, we consider nodes select for overhearing the highest degree channel from their ECS list only. If more than one option is available, a random choice for transmission/overhearing is performed among those channels with the same degree.

Since, our goal is to efficiently disseminate the data, tuning of sender and receiver nodes to the same channel with high probability, and to protect the PR nodes from harmful interference, we define five performance metrics:

1. Harmful Interference Ratio (HIR): This metric is defined in order to capture the notion of collision with PR nodes. HIR is defined as the ratio of the total number of times the channel is occupied by PR node after the channel selection decision over total number of times the channel selection decision occurs.

2. Average Delivery Ratio: This metric is defined to effectively measure the data dissemination process. It is the ratio of packets received by a particular CR node over total packets sent in the network.

3. Ratio of Accumulative CR Receivers: This metric also evaluates the data dissemination process. It is defined as the average ratio of accumulative $\mathrm{CR}$ receivers per hop over the accumulative effective neighbors per hop. Accumulative CR receivers per hop are the number of CR receivers per hop that successfully received the message, while accumulative effective neighbors per hop are the CR neighbors that selects the same channel for overhearing as the sender node used for transmission. Note that by accumulative ratio we mean: at each new hop $n$, the receivers and effective neighbors of all previous hops $l<n$ are summed up to the ones at hop $n$.

4. Ratio of Effective Neighbors and Ratio of Accumulative Effective Neighbors: Ratio of effective neighbors are the number of neighbors that selects the same channel for overhearing as the sender node used for transmission over the total number of CR neighbors. While, Ratio of accumulative effective neighbors are the effective neighbors of all previous hops $l<n$ are summed up to the ones at hop $n$ over the total number of CR neighbors of all previous hops $l<n$ are summed up to the ones at hop $n$.

\subsection{Simulation Environment}

The transmission range of CR nodes is set to $R=250 \mathrm{~m}$. The number of CR nodes is fixed to $\mathrm{N}=100$ and CRs are randomly deployed within a square area of $a^{2}=$ $700 \times 700 m^{2}$. Simulations run for 1000 seconds. Total 1000 packets were sent, where each packet is sent by a randomly selected node after 1 second. All results are obtained with a confidence interval of $95 \%$.

We consider $5(C h=5)$ and $10(C h=10)$ total number of channels, which allows varying the neighborhood density $d_{\text {avg }}$ between 11.3 (when $\mathrm{Ch}=5$ ) and 20.1 (when 
Table 2: Wireless channel parameters used in the simulation.

\begin{tabular}{c|c|c|c|c|c|c|c|c|c|c}
\hline & Ch 1 & Ch 2 & Ch 3 & Ch 4 & Ch 5 & Ch 6 & Ch 7 & Ch 8 & Ch 9 & Ch 10 \\
\hline \hline$\lambda_{X}$ & 1.25 & 0.4 & 1 & 0.4 & 0.5 & 2 & 1 & 0.18 & 0.5 & 0.67 \\
\hline$\lambda_{Y}$ & 0.67 & 2 & 1 & 0.33 & 1 & 0.29 & 0.25 & 2 & 1.33 & 0.5 \\
\hline$u^{i}$ & 0.35 & 0.83 & 0.5 & 0.45 & 0.67 & 0.13 & 0.2 & 0.92 & 0.73 & 0.43 \\
\hline
\end{tabular}

Table 3: Number of retries and probability values.

\begin{tabular}{|c|c|c|c|c|c|c|c|c|}
\hline Channels & Probability & $1^{\text {st }}$ Try & $2^{\text {nd }}$ Try & $3^{\text {rd }}$ Try & $4^{\text {th }}$ Try & $5^{\text {th }}$ Try & \multicolumn{2}{|c|}{ Sum } \\
\hline \hline \multirow{2}{*}{5} & $P_{S M}$ & $49.57 \%$ & $10.9 \%$ & $3.81 \%$ & $1.94 \%$ & $1.63 \%$ & $68 \%$ & \multirow{2}{*}{$100 \%$} \\
\cline { 2 - 9 } & $P_{U M}$ & $21.6 \%$ & $6.37 \%$ & $2.3 \%$ & $1.2 \%$ & $0.5 \%$ & $32 \%$ & \\
\hline \multirow{2}{*}{10} & $P_{S M}$ & $58 \%$ & $9.4 \%$ & $1.6 \%$ & $0.5 \%$ & $0.2 \%$ & $70 \%$ & \multirow{2}{*}{$100 \%$} \\
\cline { 2 - 8 } & $P_{U M}$ & $22.5 \%$ & $4.9 \%$ & $1.69 \%$ & $0.7 \%$ & $0.2 \%$ & $30 \%$ & \\
\hline
\end{tabular}

$\mathrm{Ch}=10$ ). Note this density is computed after the spectrum sensing provides the list of available channels and before the CRs select the channel to transmit/overhear. In this case, it is worth mentioning that, at the following simulation studies, the neighborhood density varies in function of the CRs' channel selection and is lower than the above ones.

$T T L$ is introduced to disseminate the message in the whole network. It is the maximum number of hops required for a packet to traverse the whole network, i.e., $\left\lceil\frac{2 a}{R}\right\rceil$, and is set to $T T L=6$ in our simulation scenario. Details on the used wireless channel parameters (rate of exponential distribution i.e., $\lambda_{X}$ and $\lambda_{Y}$ ) can be found in Table. 2, which were measured by authors in [29]. These rate values can be easily measured from the sample of the number of transitions (ON to OFF, OFF to ON, ON to ON, and OFF to OFF) a channel follows, as mentioned in [29].

In summary, at each packet transmission event, the PR unoccupancy per channel $i,\left(P R_{u}^{(i)}\right)$, is calculated by each CR node. Then, each CR node locally computes the CR occupancy $\left(C R_{o}^{(i)}\right)$ and the weight $\left(P_{w}^{(i)}\right)$ of each channel $i$. The channel with the highest weight is then selected for transmission and/or overhearing. The message dissemination phase then starts, in which a randomly selected CR node disseminates the message on the selected channel by setting a TTL at the message. CR neighbor nodes that are on the same channel will overhear the message, decrease the $T T L$, redo the spectrum sensing, select the best available channel, and disseminate the message to the next-hop neighbors until $T T L=0$.

In the following section, we perform comprehensive analysis of SURF. We first evaluate different parameters related to SURF in section 7.4. Then in section 7.5, we discuss and evaluate SURF by comparing it with three related approaches.

\subsection{SURF Parameters Evaluation}

We have defined $P_{S M}$ as the probability of successfully matched state, and $P_{U M}$ as the probability of unsuccessfully matched state, in Section 5.1. Moreover, we have also mentioned the number of retries by SURF when the channel in occupied in Section 4. In this section, our goal is to evaluate and understand them. 


\subsubsection{Retries in SURF}

In this section, we evaluate different probabilities, such as $P_{S M}, P_{U M}, P_{M D}$, and $P_{F A}$ (cf. section 5.1) and the number of retries by SURF. The number of retries means that when the channel has high weight but at time $t$ it is occupied, SURF reacts by selecting the next best weighted channel for packet transmission.

Fig. 4 shows the ratio of $P_{S M}, P_{U M}, P_{M D}$, and $P_{F A}$ states and number of retries in SURF, when $C h=5$ and $C h=10$. Where $P_{S M}$ is the probability of successfully matched state, $P_{U M}$ is the probability of unsuccessfully matched state, $P_{M D}$ is probability of miss-detection, and $P_{F A}$ is probability of false-alarm. Note that the sum of all the retries gives $P_{S M}+P_{U M}=1$ and $P_{U M}=P_{M D}+P_{F A}$. When $C h=5$, the $P_{S M}$ and $P_{U M}$ values can be seen in Table. 3. The sum of $P_{S M}$ for all the retries is $68 \%$ and the sum of $P_{U M}$ for all the retries is $32 \%$. Hence, the sum of $P_{S M}+P_{U M}=68 \%+32 \%=100 \%$ for $C h=5$. In the same manner, when $C h=10$, the $P_{S M}$ and $P_{U M}$ values are also shown in Table. 3. The sum of $P_{S M}$ for all the retries is $70 \%$ and the sum of $P_{U M}$ for all the retries is $30 \%$. Hence, the sum of $P_{S M}+P_{U M}=70 \%+30 \%=100 \%$ for $C h=10$.

At the $1^{\text {st }}$-try, when $C h=5, \mathrm{SURF}$ has the ratio of $49.57 \%$ of $P_{S M}$, and $21.6 \%$ of $P_{U M}$. But when the number of channels increase to 10 i.e., $C h=10$, SURF has higher ratio of $P_{S M}$ i.e., $P_{S M}=58 \%$, and the ratio of $P_{U M}$ is $22.5 \%$. This is due to the fact that a lower number of channels also reduce the chances for CR nodes finding PR-unoccupied channels for their transmission. When $C h=10$, the ratio of $P_{S M}$ is $9.4 \%$ at the $2^{n d}$-retry and the number of retries decrease and as we can see in the figure that at the $5^{t h}$-retry, the ratio is almost $0.2 \%$. This clearly shows that SURF is able to find unoccupied channels at the $1^{\text {st }}$ and $2^{\text {nd }}$ tries.

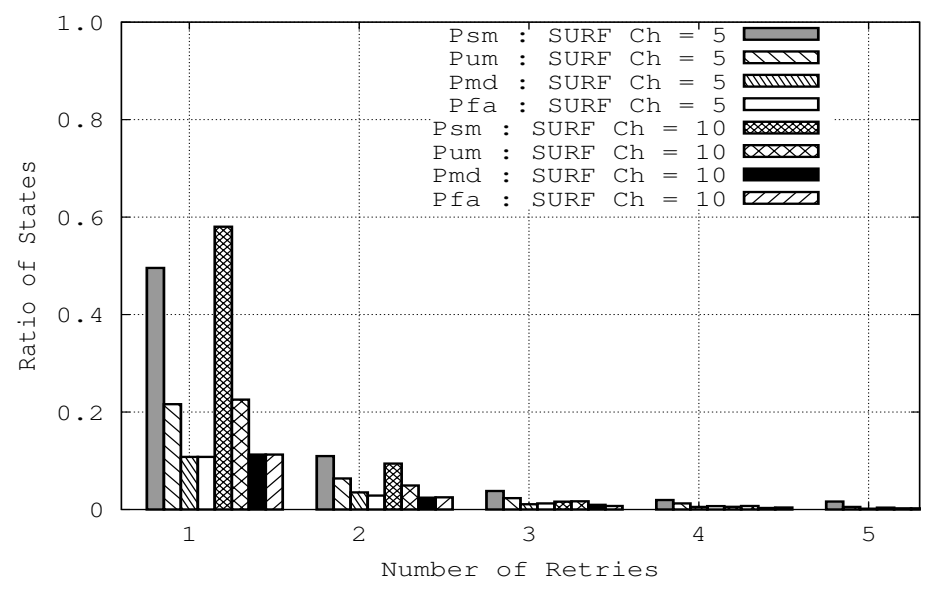

Figure 4: Ratio of $P_{S M}, P_{U M}, P_{M D}$, and $P_{F A}$ states and number of retries in SURF.

\subsubsection{Impact of Varying Neighborhood Density on SURF}

To evaluate the impact of varying neighborhood density on SURF, we vary the average neighborhood density $d_{\text {avg }}$ from 11.3 to 15.0 (when $\mathrm{Ch}=5$ ) and from 20.1 to 26.8 (when 


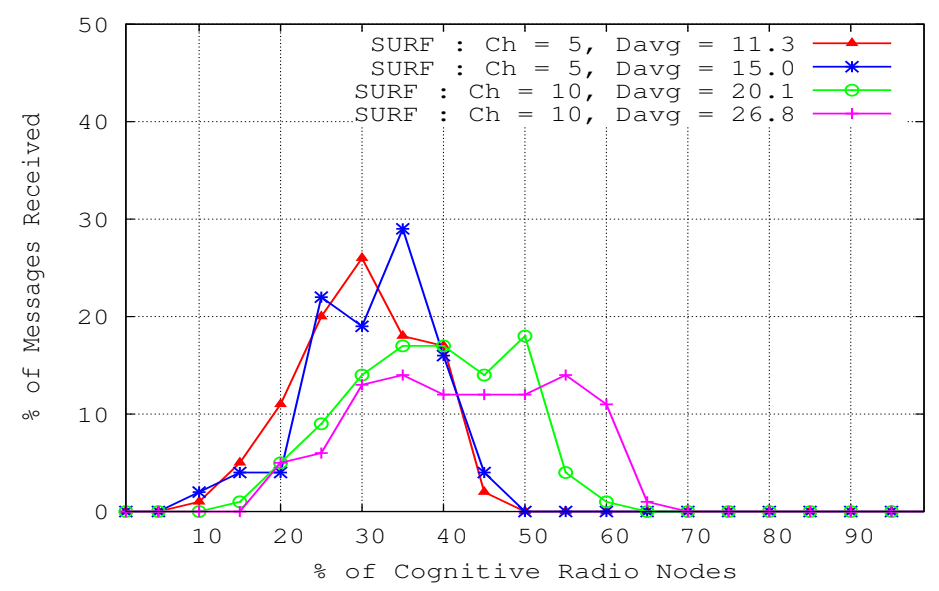

Figure 5: Percentage of Messages received by percentage of CR nodes in SURF under varying node density.

$\mathrm{Ch}=10$ ). In order to achieve this neighborhood density, we fixed the transmission range of CR nodes to $R=250 \mathrm{~m}$ and reduce the size of the network from $a^{2}=700 \times 700 \mathrm{~m}^{2}$ to $a^{2}=600 \times 600 m^{2}$. Since, the size of the network decreases, therefore $T T L$ becomes $T T L=5$.

Fig. 5 shows the percentage of messages received by percentage of CR nodes in SURF, under varying neighborhood density. SURF increases the reachability of CR nodes with the increase of average neighborhood density. For e.g., when $C h=10$ and the average node density is $26.8,60 \%$ of CR nodes received $10 \%$ of messages, while when the average node density is 20.1 , only $2 \%$ of messages were received by $60 \%$ of nodes. This is due to the fact that when node density is low, CR nodes receives high number of messages because of less collisions but the message does not propagate fully in the network. When node density is high, CR nodes receives low number of messages because of high collisions but a small percentage of messages are still able to be propagated in the network and thus, SURF is able to increase the reachability of CR nodes under higher node density.

\subsubsection{PR Utilization of the Selected Channel}

As mentioned earlier that when the channel has high weight but at time $t$ it is occupied, SURF reacts in this case (i) by not transmitting the packet on the best weighted channel and (ii) by selecting the next best weighted channel for packet transmission/overhearing. Also note that when all the channels are occupied, no message is sent. We now evaluate SURF by looking at the PR utilization of the selected channel and the number of retry for sent message. PR utilization means the PR activity on the selected channel. Note that there were 1000 total messages sent. In Fig. 6 and Fig. 7, we plot for each sent message, the retry and the PR utilization of the selected channel by SURF.

Fig. 6 and Fig. 7 shows the PR utilization of the selected channel by SURF, when $C h=5$ and $C h=10$, respectively. When $C h=5$ (cf. Fig. 6), we see that the majority of times SURF selects the channel at the $1^{\text {st }}$ try and the PR utilization of the 
selected channel is also low. But there are some rare cases when SURF selects more PR occupied channels and we can also see that there are very few cases when SURF goes to $3^{r d}$ and $4^{t h}$ retry. This is due to the fact that a lower number of channels also reduce the chances for CR nodes finding PR-unoccupied channels for their transmission. But when the number of channels increase to 10 , i.e., $C h=10$, SURF selects the least PR utilized channel and all the messages sent almost at the $1^{\text {st }}$ and $2^{\text {nd }}$ retries.

SURF : Ch $=5$, PR Utilization $=$

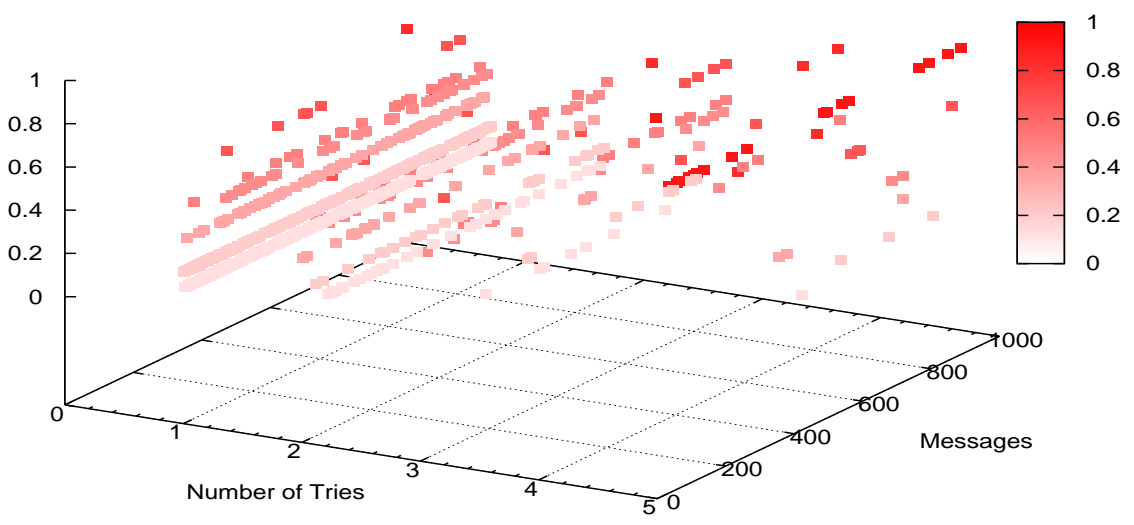

Figure 6: Sent Messages, Retries, and the PR utilization of the selected channel in

SURF, when $\mathrm{Ch}=5$.

\subsection{SURF Comparison}

In this section, we evaluate the performance of SURF by comparing it with three related approaches i.e., Random (RD), Highest Degree (HD), and Selective Broadcasting (SB).

\subsubsection{Protection to Primary Radio Nodes}

In this section, we characterize the probable interference caused by CR transmissions to PR nodes for SURF, RD, HD, and SB. Fig. 8 compares the harmful interference ratio for the four strategies i.e. $\mathrm{RD}, \mathrm{HD}, \mathrm{SB}$ and $\mathrm{SURF}$, for $\mathrm{Ch}=5$ and $\mathrm{Ch}=10$. It can be clearly seen in the figure that SURF, as expected, causes less harmful interference to $\mathrm{PR}$ nodes, compared to $\mathrm{RD}, \mathrm{HD}$, and $\mathrm{SB}$. This is primarily because, when using SURF, CR nodes select those channels that have very high probability of being in OFF state, reducing thus PR interference. Note that in SURF, if all channels are occupied, the CR transmission will not take place. Thus, the lower HIR value for SURF in Fig. 8 is shown only to represent the cases where all channels were occupied by PRs and a 


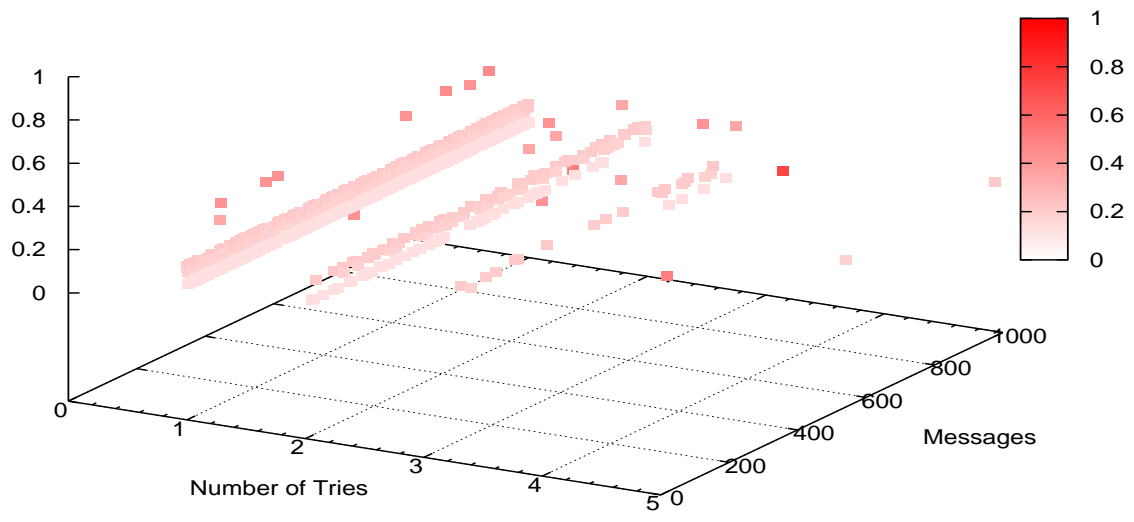

Figure 7: Sent Messages, Retries, and the PR utilization of the selected channel in SURF, when $\mathrm{Ch}=10$.

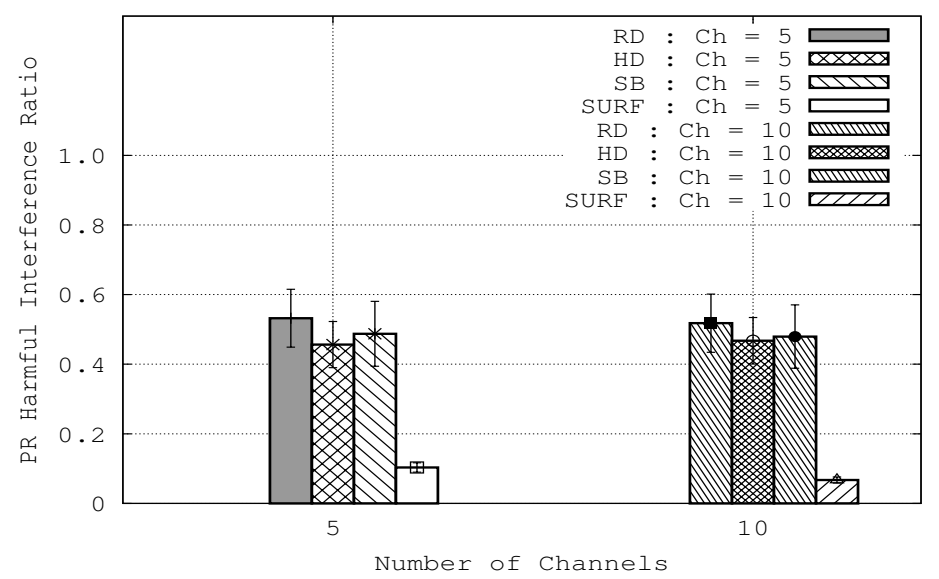

Figure 8: PR harmful interference ratio for RD, HD, SB and SURF, when $\mathrm{Ch}=5$ and $\mathrm{Ch}=10$. 
probable interference would be caused if a transmission took place. In addition, when the number of channels is low, i.e. $\mathrm{Ch}=5$, the value of HIR is higher than $\mathrm{Ch}=10$. This is due to the fact that a lower number of channels also reduce the chances for CR nodes finding PR-unoccupied channels for their transmission. As a result, SURF protects PR nodes, by reducing the amount of collisions with primary radios.



Figure 9: CR Nodes' ID and average delivery ratio.

\subsubsection{Reliable Data Dissemination}

In this section, our goal is to evaluate the reliability of data dissemination. We have chosen two parameters to evaluate reliable data dissemination: (1) average delivery ratio, and (2) ratio of accumulative receivers.

Average Delivery Ratio: Fig. 9 compares the average delivery ratio of RD, HD, SB and SURF, for $\mathrm{Ch}=5$ and $\mathrm{Ch}=10$. SURF increases considerably the delivery ratio compared to the other solutions. In particular, for $\mathrm{Ch}=5, \mathrm{SURF}$ guarantees the delivery ratio of approximately $20 \%-30 \%$ compared to almost $0 \%$ in the case of $\mathrm{RD}, \mathrm{HD}$, and $\mathrm{SB}$. And when $\mathrm{Ch}=10$, the average delivery ratio of SURF is almost $40 \%-50 \%$, while in $\mathrm{RD}$, it is almost $0 \%, 2 \%$ in HD and $1 \%$ for SB. In fact, RD, HD, and SB, do not guarantee that the selected channel is unoccupied for transmission thus causing a severe decrease in the delivery ratio. While in SURF, the average delivery ratio is higher because CR nodes select the channel that has higher $P_{O F F}^{*}(t)$ and higher CR neighbors. It is worth mentioning that the diversity in terms of available channels and of PR activities as well as the consequent lower neighborhood density after CRs local channel selection result in the creation of different topologies (i.e., dynamic neighborhood) at each transmission/overhearing of CR nodes. These issues make hard the achievement of a higher delivery ratio than SURF. Most importantly, it is worth noting that with the increase of the number of channels, SURF performance is also enhanced. This result is counterintuitive since adding more channels makes the synchronization between the sender and the receiver (i.e selecting the same channel) harder to achieve. However, by using the appropriate metric and mainly employing the same strategy at the sender and 




Figure 10: Hop count and Ratio of accumulative receivers.

the receiver, SURF achieves better results when more channels are available. This is also due to the fact that with more channels, PR activity over the channels decreases.

Ratio of Accumulative Receivers: Fig. 10 compares the ratio of accumulative receivers at each hop of communication (i.e., until $T T L=0$ ) for RD, HD, SB, and SURF. SURF outperforms the three other techniques in all hops. At the $1^{\text {st }}$-Hop, due to the first transmission of the message, no collision is present. In this case, SURF provides a ratio of $95 \%$ receivers for $\mathrm{Ch}=10$ ( $80 \%$ for $\mathrm{Ch}=5)$, against $5 \%$ for $\mathrm{RD}, 12 \%$ for $\mathrm{HD}$, and $2 \%$ for SB. With the message propagation and its natural replication in the network, the probability of collisions increases and consequently, the receivers' ratio at each new hop decreases, for all the strategies. Still, SURF provides a better dissemination ratio than other strategies. This is obtained thanks to the SURF channel selection, which selects channels providing high probability for good delivery as well as for good reception.

In summary, results in Fig. 9 and Fig. 10 confirm that SURF can provide good network reachability, suitable for increasing dissemination reliability in multi-hop cognitive radio ad-hoc networks.

\subsubsection{Tuning of Sender and Receiver}

In this section, we evaluate and characterize the tuning of sender/receiver nodes. We have defined two metrics: (1) ratio of average effective neighbors, and (2) ratio of average accumulative effective neighbors (cf. section 7.2).

Fig. 11 compares the ratio of average effective neighbors over the total average number of $\mathrm{CR}$ neighbors of RD, HD, SB and SURF, for $\mathrm{Ch}=5$ and $\mathrm{Ch}=10$. SURF has higher ratio of effective neighbors compared to $\mathrm{RD}$ and $\mathrm{SB}$, while almost equal ratio of effective neighbors to HD. This is primarily because SURF and HD prefer to select those channels that have higher number of neighbors. Since, SURF also consider PR unoccupancy (cf. Fig. 8), therefore, majority of transmission are successful, which is not the case in HD (cf. Fig. 10). Moreover, this also results in the decrease of the delivery ratio and the ratio of accumulative receivers (cf. Fig. 9 and Fig. 10). This 
justify that SURF is able to tune both sender and receiver to the right channel with high probability for effective and reliable data dissemination in multi-hop context.

Fig. 12 compares the ratio of average accumulative effective neighbors over the average accumulative total CR neighbors of RD, HD, SB and SURF, for $\mathrm{Ch}=5$ and $\mathrm{Ch}=10$. It is worth mentioning that $\mathrm{CRs}$ local channel selection result in the creation of different topologies (i.e., dynamic neighborhood) at each transmission/overhearing of CR nodes. As can be clearly seen in the figure that at the $6^{t h}-H o p$, RD is only able to create a connected topology of $20 \%(C h=5)$ and $9 \%(C h=10)$ nodes in the network. SB is able to create a topology of $27 \%(C h=5)$ and $32 \%(C h=10)$ nodes in the network. HD is able to create a connected topology of $63 \%(C h=5)$ and $54 \%$ $(C h=10)$, while SURF is able to create a connected topology of $60 \%(C h=5)$ and $56 \%(C h=10)$ nodes in the network.



Figure 11: Ratio of Average Number of Effective Neighbors for RD, HD, SB and SURF.

\subsubsection{Packet Ratio}

We now analyze the performance of RD, HD, SB, and SURF by evaluating the packet ratio of different types, e.g., received, missed, and interrupted packet ratio. We measure the packet ratio in single-hop context and multiple-sources are considered throughout the network. Table 4 shows packet ratio description used in the simulation. The received packet ratio is used to quantify the data dissemination success, missed packet ratio is used to quantify the packet losses due to nodes overhearing on different channel, while interrupted packet ratio is used to quantify the harmful interference to PR nodes.

Fig. 13 and Fig. 14 compares the packet ratio of RD, HD, SB and SURF, when $\mathrm{Ch}=5$ and $\mathrm{Ch}=10$, respectively. The packet received ratio of SURF is higher compared to RD, HD, and SB. This is primarily because SURF emphasis on selecting those channels that has higher number of neighbors. Conversely, due to the same reason, an opposite behavior can be seen in the packet missed ratio i.e. the packet missed ratio of SURF is lower than RD and HD. Note that when the number of channels increase from 


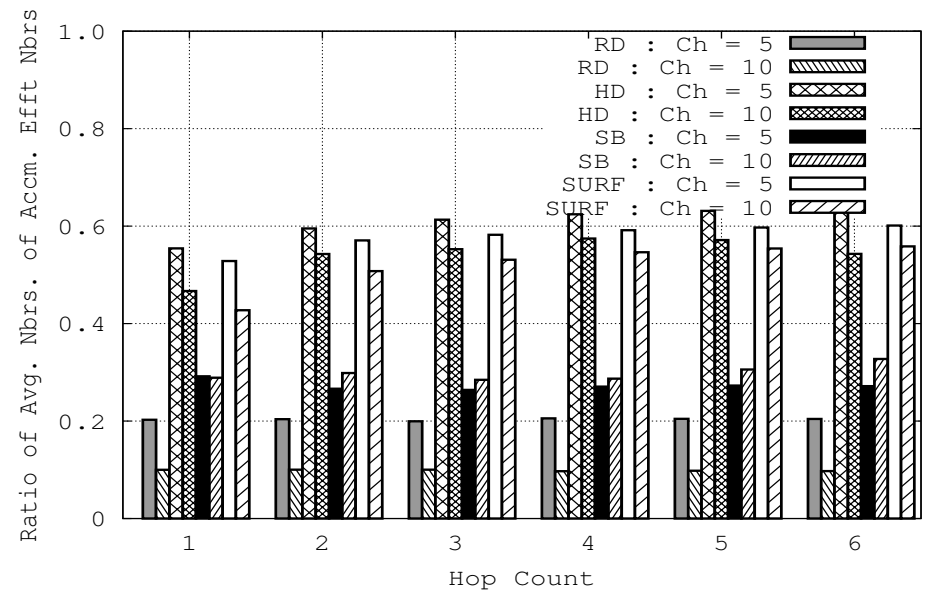

Figure 12: Ratio of Average Number of Accumulative Effective Neighbors for RD,

HD, SB and SURF.

$\mathrm{Ch}=5$ to $\mathrm{Ch}=10$, the missed packet ratio of SB increases. This is due to the fact that when the number of channels increases in SB, the CR neighbors are spread over more channels and when the node broadcast on the channel, there are more chances that the CR neighbors drop the packet being overhearing on different channel. Both the packet received ratio and packet missed ratio reveals that SURF disseminates well the packets to the neighboring nodes, compared to $\mathrm{RD}, \mathrm{HD}$, and $\mathrm{SB}$ approaches.

The interrupted packet ratio in Fig. 13 and Fig. 14 shows that SURF drops less number of packets compared to RD, HD, and SB. This is due to the fact that SURF considers PR activity, while selecting the channel for transmission. More particularly, in SURF, the interrupted packet ratio decreases, when the number of channels increase from $C h=5$ to $C h=10$. This is primarily because when the number of channels increase, SURF has higher chances to find the PR unoccupied channels.

Table 4: Packet Ratio Description.

\begin{tabular}{c|c}
\hline Received Packets & $\begin{array}{r}\text { Ratio of the total number of nodes that received the packets } \\
\text { and total number of neighbor nodes }\end{array}$ \\
\hline Missed Packets & $\begin{array}{r}\text { Ratio of the total number of nodes that do not received the packets (due to the selection } \\
\text { of a different channel) and the total total number of neighbor nodes }\end{array}$ \\
\hline Interrupted Packets & Ratio of the total number of nodes that do not received the packets (due to PR activity) \\
and the total number of neighbor nodes
\end{tabular}




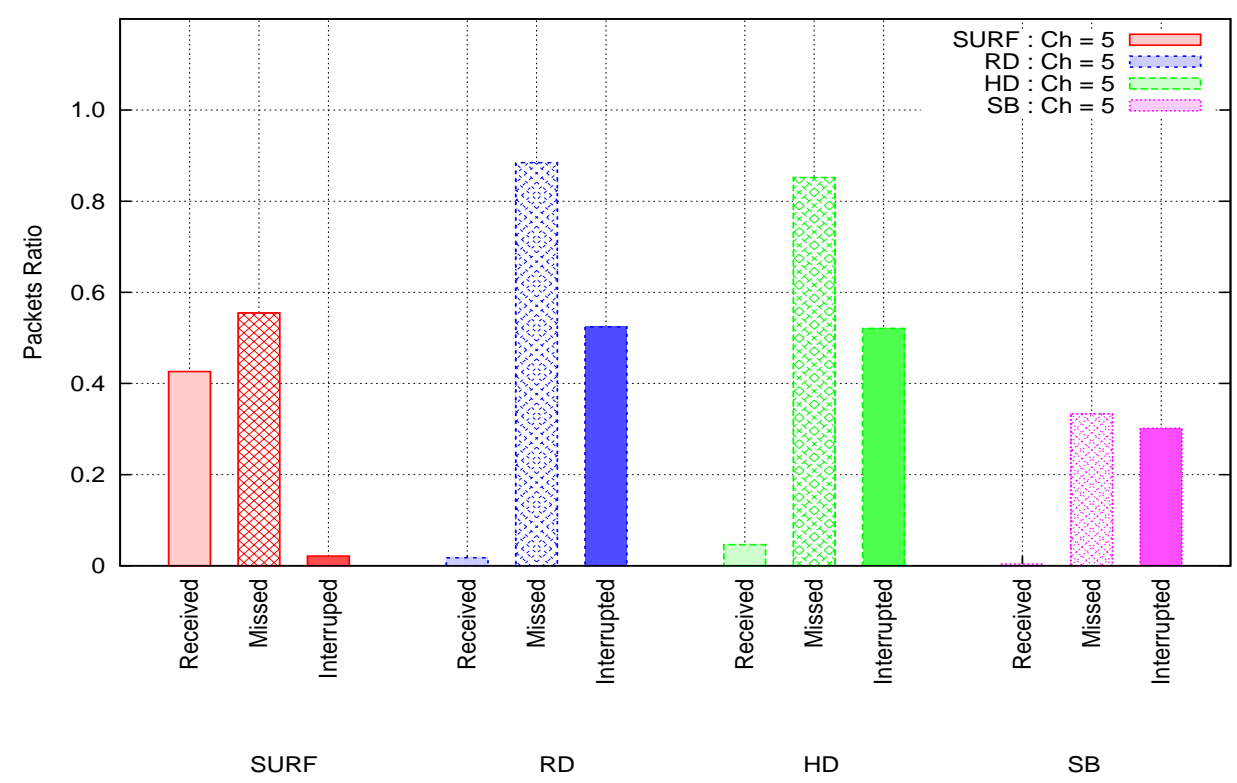

Figure 13: Packet Ratio for RD, HD, SB and SURF when $\mathrm{Ch}=5$.

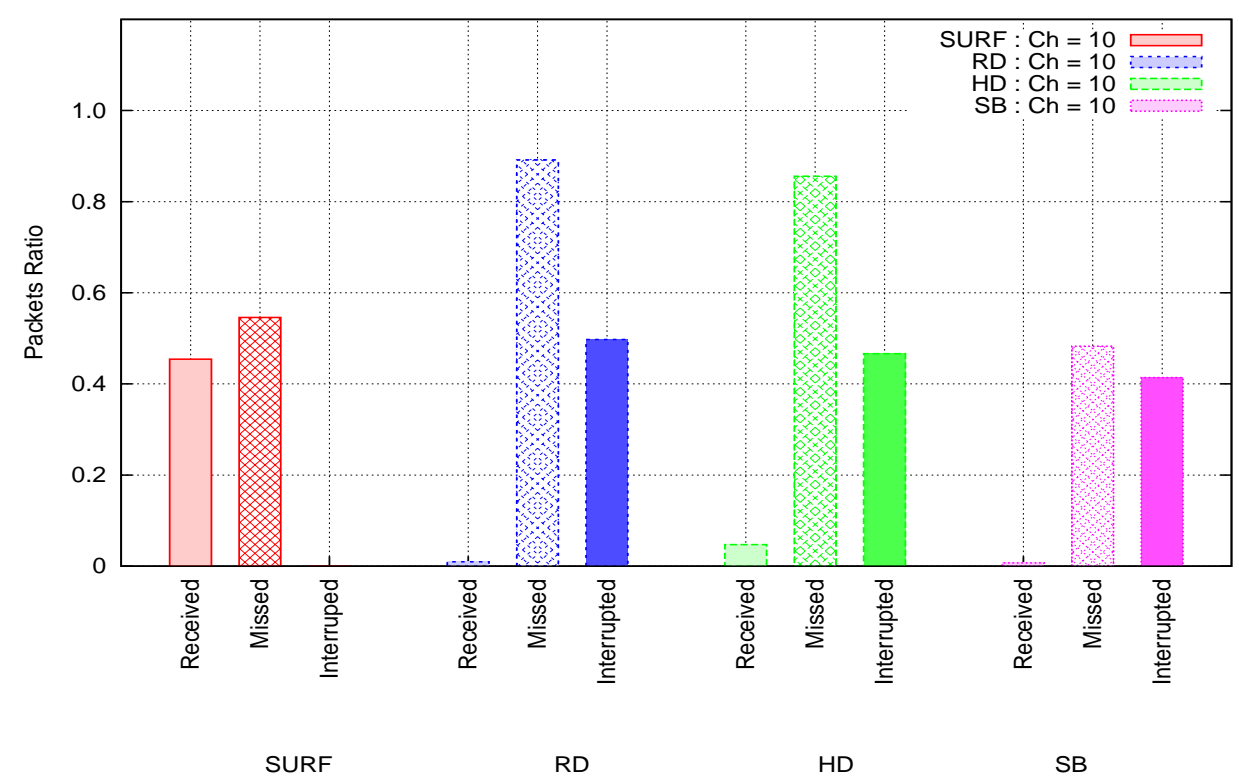

Figure 14: Packet Ratio for RD, HD, SB and SURF when $\mathrm{Ch}=10$. 


\section{Activity pattern impact of PR Nodes on Channel Se- lection Strategies}

\subsection{Context}

Cognitive radio wireless networks (CRNs) [3] are designed to use the radio spectrum opportunistically. CRNs are composed of two types of nodes: Primary Radio (PR) nodes and the Cognitive Radio (CR) nodes. Primary radio nodes are the legacy users and they have the high priority to use the channels for communication. However, cognitive radio nodes can only use the channels when they are idle i.e. not utilized by the PR nodes. Therefore, the performance of cognitive radio network is highly dependent upon the primary radio nodes activity pattern. The primary radio nodes activity pattern i.e. presence or absence of the PR signal, can be modelled as continuous-time, alternating ON/OFF Markov Renewal Process (MRP) [26, 27, 28]. This PR activity model has been used very widely in the literature [26, 27, 28].

Recently, very few works has been done to analyze PR nodes activity pattern. In [43], the authors model and evaluate the performance of Transmission Control Protocol over Cognitive Radio Ad Hoc Networks. The authors considered a single-hop topology for PR activity analysis and four different regions (long term, high, low, intermittent) for PR nodes activity. The effect of PR ON/OFF periods on the system performance in the context of MAC protocol is evaluated in [44]. In [45], the authors studied the influence of the activity patterns of the primary radio transmitters on the area in which cognitive radios have opportunities for spectrum reuse, with the given transmit power. But none of these works have analyzed the impact of different PR nodes activity pattern on different channel selection strategies as well as on data dissemination. Moreover, these works do not consider the effect of PR nodes activity in a multi-hop network. In fact, due to lack of centralized entity and the difficult coordination between CR nodes in multi-hop cognitive radio ad-hoc network, the selection of a common channel by $\mathrm{CR}$ transmitters and receivers is a challenging task.

\subsection{Channel Selection Strategies}

In this section, we first describe the channel selection strategies that we consider in our analysis.

We consider a RD approach in which channels are randomly selected to be used by $\mathrm{CR}$ nodes for transmission and/or overhearing, without any consideration to the ongoing PR and CR activity over these channels. HD approach only considers CR activities and is inspired by SB approach. In HD, CR nodes select the highest CR degree channel for transmission and overhearing, without any consideration of PR activity. The highest degree channel covers, consequently, the highest number of neighbors in the available list of channels.

In SB [17], each CR node calculates a minimum set of channels, Essential Channel Set (ECS), for transmission that covers all its geographic neighbors, without considering the PR unoccupancy. In SB, a CR node transmits on multiple channels in roundrobin fashion present in the ECS list, until all neighbors are covered. Note that in [17] nothing is mentioned about how nodes overhear over the channels. Therefore, we consider nodes select for overhearing the highest degree channel from their ECS list only. 
If more than one option is available, a random choice for transmission/overhearing is performed among those channels with the same degree.

SURF [46] is our distributed channel selection strategy specifically designed for data dissemination in multi-hop cognitive radio networks. In SURF, special consideration is given to select those channels that cause less harmful interference to PR nodes. This is achieved by considering the PR nodes activity pattern during the channel selection decision. On top of that, SURF gives high preference to those channels that have higher number of CR neighbors.

SURF strategy classifies channels by assigning a weight $P_{w}^{(i)}$ to each observed channel $i$ in the channel set $C$. Thus, every cognitive radio running SURF, locally computes the $P_{w}^{(i)}$ using the following equation:

$$
\forall i \in C: P_{w}^{(i)}=P R_{u}^{(i)} \times C R_{o}^{(i)}
$$

$P_{w}^{(i)}$ describes the weight of a channel $i$ and is calculated based on the unoccupancy of PR (i.e. $P R_{u}^{(i)}$ ) and CR occupancy (i.e. $C R_{o}^{(i)}$, which reflects the number of CR neighbors) over channel $i$. Then, the channels are ranked according to their weights and the best channel (i.e., the one providing highest $P_{w}^{(i)}$ ) is selected. SURF has also the mechanism of recovery from bad channel selection decision, which is considered during the computation of the primary radio unoccupancy. In this mechanism, SURF keeps track of previous wrong channel state estimation and accordingly adapts future channel selection decision. The primary radio unoccupancy $P R_{u}^{(i)}$ is given by:

$$
P R_{u}^{(i)}=P_{O F F}^{*}(t)^{(i)}=P_{O F F}^{(i)}\left(1-P_{F A}^{(i)}\right)+P_{M D}^{(i)}\left(1-P_{O F F}^{(i)}\right)
$$

where $P_{O F F}(t)$ (cf. Eq. 13) is the probability that the channel $i$ will be in OFF state at time $t, P_{F A}^{(i)}$ is the probability of false alarm, and $P_{M D}^{(i)}$ is the probability of miss-detection. More details on how these probabilities are calculated can be found in our paper [46] and in section 5 .

\subsection{Primary Radio Nodes Activity Pattern}

The primary radio nodes activity, i.e. presence or absence of the PR signal, can be modelled as continuous-time, alternating ON/OFF Markov Renewal Process (MRP) [26,27, $28]$. This PR activity model has been used very widely in the literature $[26,27,28]$. The ON/OFF PR activity model approximates the spectrum usage pattern of public safety bands [36]. The public safety band is designated for commercial and public safety uses [37]. The authors in [38] approximate and validate the PR ON/OFF activity model for the presence of the PR signal in IEEE 802.11b. The ON/OFF PR activity model is also the most famous model for voice [39]. An important feature of this ON/OFF PR activity model is that it captures the time period in which the channel can be utilized by CRs without causing any harmful interference to PR nodes [29].

In this paper, we use the formulation of $[26,28,29]$ that the channels $\mathrm{ON}$ and OFF periods are both exponentially distributed with p.d.f. $f_{X}(t)=\lambda_{X} \times e^{-\lambda_{X} t}$ for ON state and $f_{Y}(t)=\lambda_{Y} \times e^{-\lambda_{Y} t}$ for OFF state. The probability that the channel $i$ will be in OFF state at time $t$, i.e., $P_{O F F}(t)$, is calculated as:

$$
P_{O F F}(t)=\frac{\lambda_{X}}{\lambda_{X}+\lambda_{Y}}+\frac{\lambda_{Y}}{\lambda_{X}+\lambda_{Y}} e^{-\left(\lambda_{X}+\lambda_{Y}\right) t}
$$

where $\lambda_{X}$ and $\lambda_{Y}$ are the rate parameter for exponential distribution.

We consider then four different PR nodes activity patterns [43,44], described as follows (see Fig. 15): 


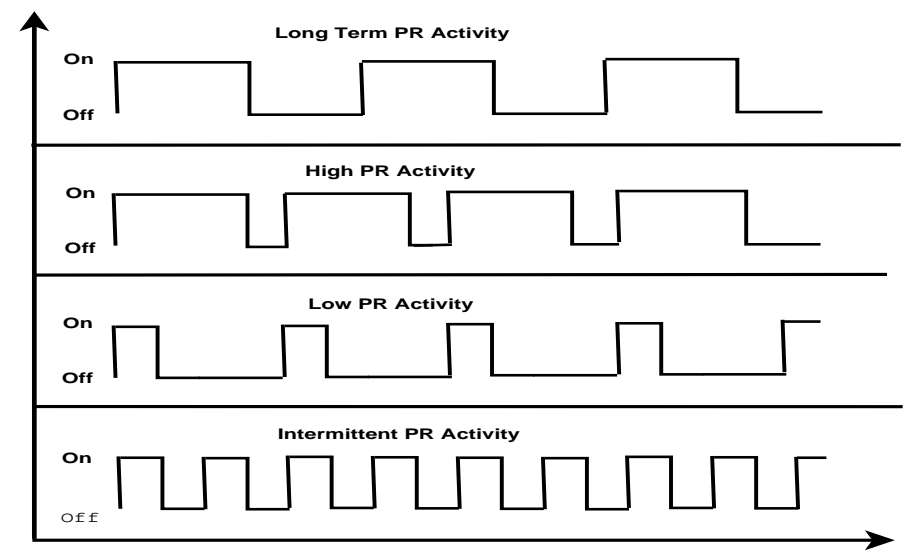

Figure 15: Long term, high, low and intermittent PR nodes activity.

- Long Term PR Activity: In Long Term PR Activity, the channel has long ON and long OFF periods. This type of PR activity can be seen in the scenarios where primary radio nodes subscribed to free call packages.

- High PR Activity: In High PR Activity, the channel has long ON and short OFF periods. This type of PR activity can be seen in highly congested urban environments or in rush hours, where all the channels are mostly occupied.

- Low PR Activity: In Low PR Activity, the channel has short ON and long OFF periods. This type of PR activity can be observed in remote areas or during less peak hours.

- Intermittent PR Activity: In Intermittent PR Activity, the channel has short ON and short OFF periods. This type of PR activity can be observed where users use the channels for very short period of time, e.g., bus stations, railway stations etc.

Fig. 15 depicts an example of these four activity patterns. In order to achieve such PR nodes activity, we vary the rate parameter $\lambda_{X}$ and $\lambda_{Y}$ of the exponential distribution, as indicated in Table $5[43,44]$.

Table 5: Primary Radio Activity.

\begin{tabular}{|c|c|c|c|c|}
\hline PR Activity & ON & OFF & $\lambda_{X}$ & $\lambda_{Y}$ \\
\hline \hline Long Term Activity & $\lambda_{X} \leq 1$ & $\lambda_{Y} \leq 1$ & Long ON & Long OFF \\
\hline High Activity & $\lambda_{X} \leq 1$ & $\lambda_{Y}>1$ & Long ON & Short OFF \\
\hline Low Activity & $\lambda_{X}>1$ & $\lambda_{Y} \leq 1$ & Short ON & Long OFF \\
\hline Intermittent Activity & $\lambda_{X}>1$ & $\lambda_{Y}>1$ & Short ON & Short OFF \\
\hline
\end{tabular}




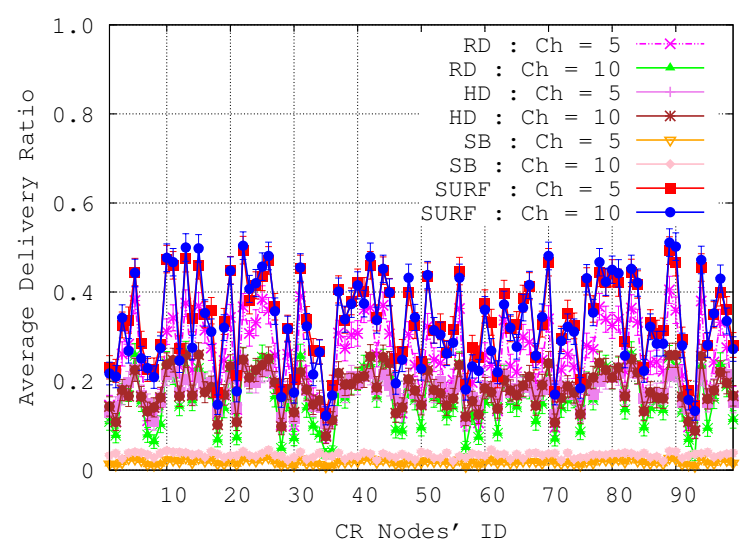

(a)

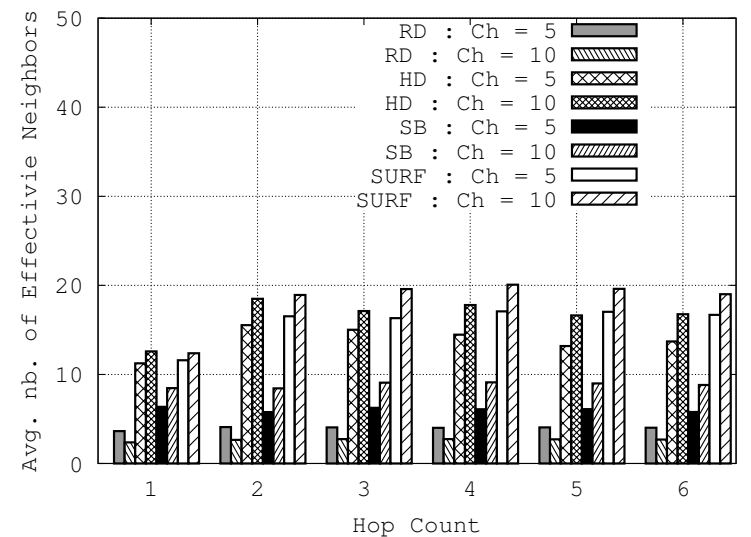

(b)

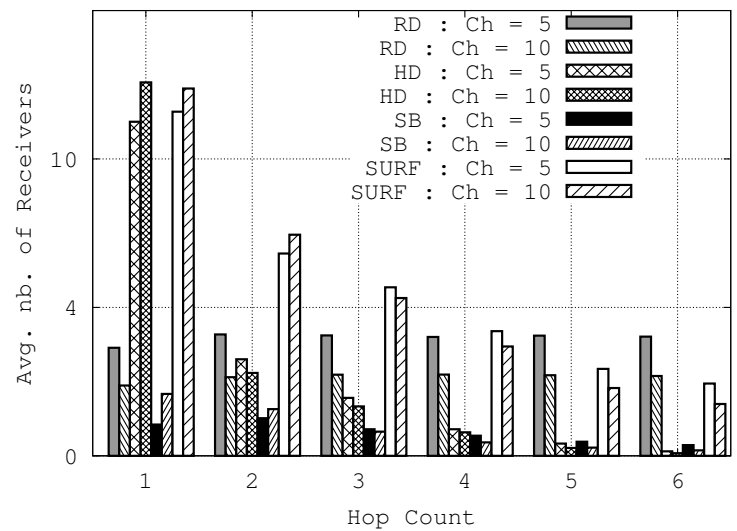

(c)

Figure 16: Zero Primary Radio Activity. (a) CR Nodes' ID and average delivery ratio for RD, HD, SB and SURF. (b) Hop count and average number of effective neighbors for RD, HD, SB and SURF. (c) Hop count and average number of receivers for RD, $\mathrm{HD}, \mathrm{SB}$ and SURF. 


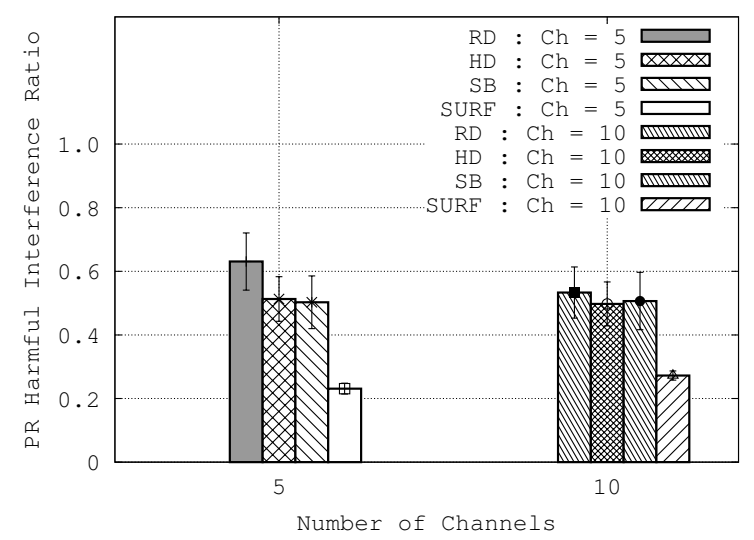

(a)

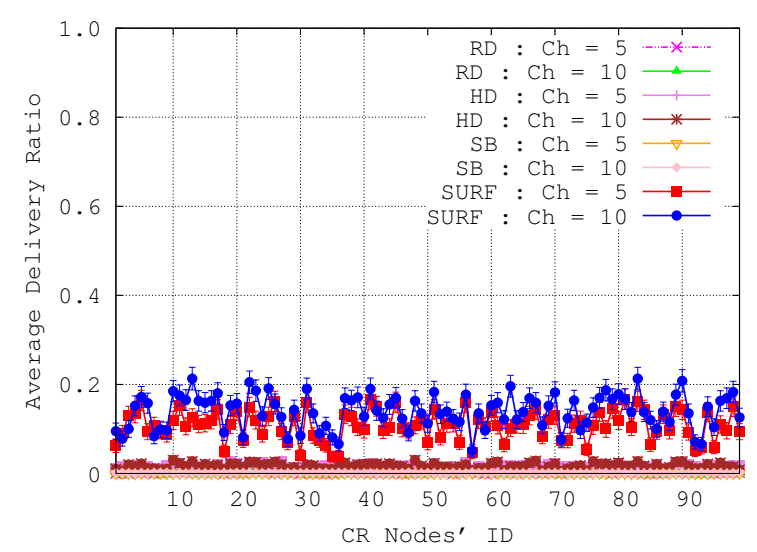

(b)

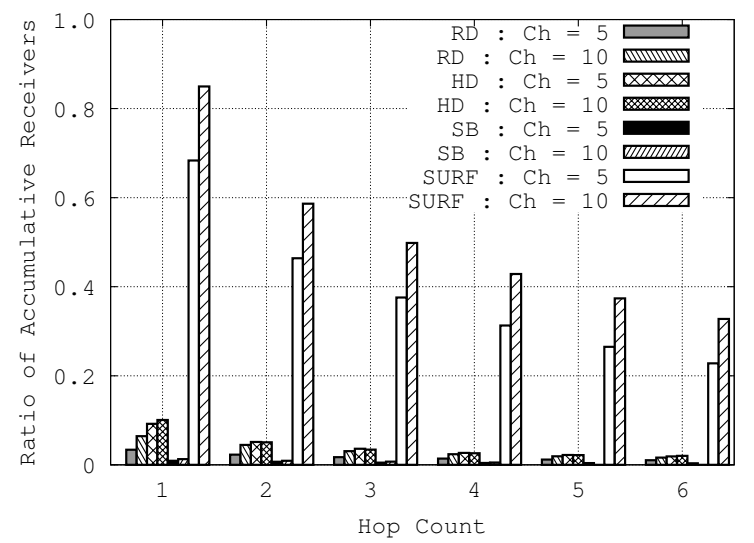

(c)

Figure 17: Long Term Primary Radio Activity. (a) PR harmful interference ratio for RD, HD, SB and SURF. (b) CR Nodes' ID and average delivery ratio for RD, HD, SB and SURF. (c) Hop count and Ratio of accumulative receivers for RD, HD, SB and SURF. 


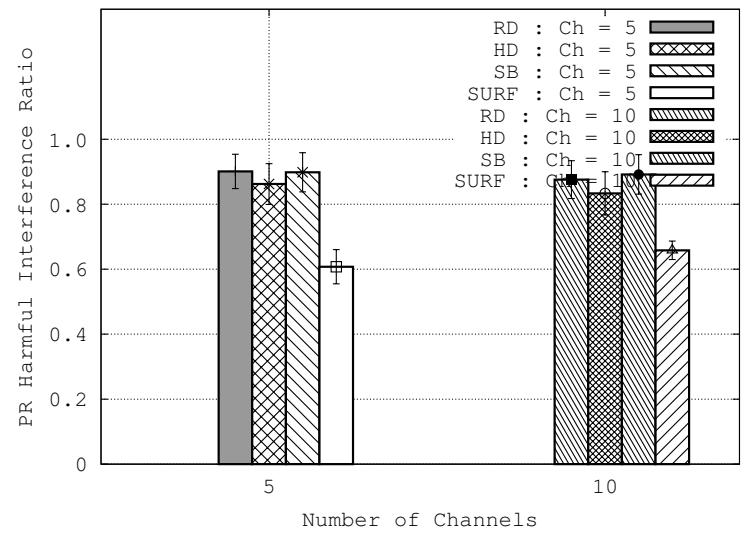

(a)

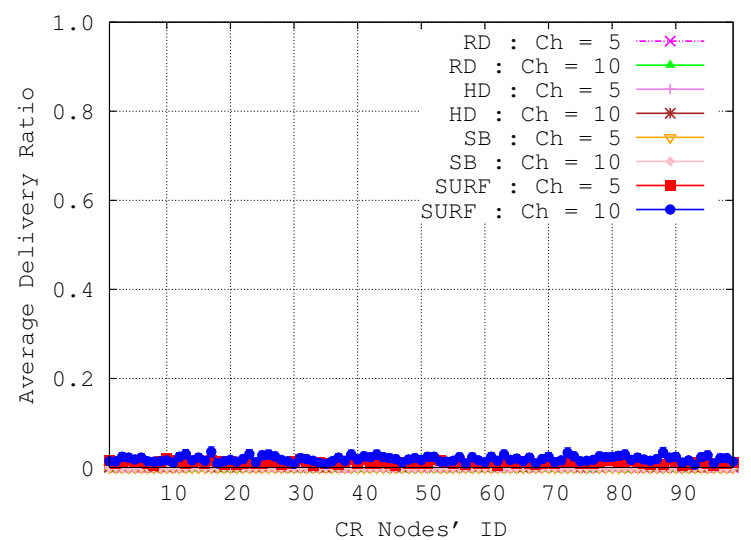

(b)

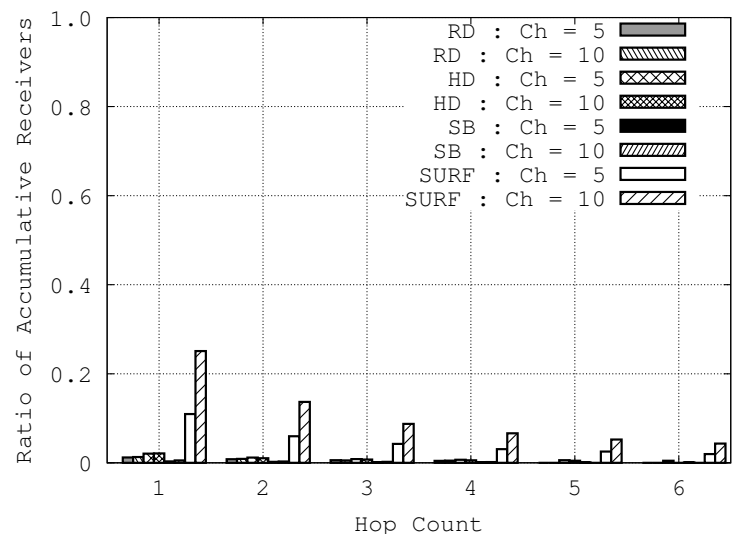

(c)

Figure 18: High Primary Radio Activity. (a) PR harmful interference ratio for RD, HD, SB and SURF. (b) CR Nodes' ID and average delivery ratio for RD, HD, SB and SURF. (c) Hop count and Ratio of accumulative receivers for RD, HD, SB and SURF. 


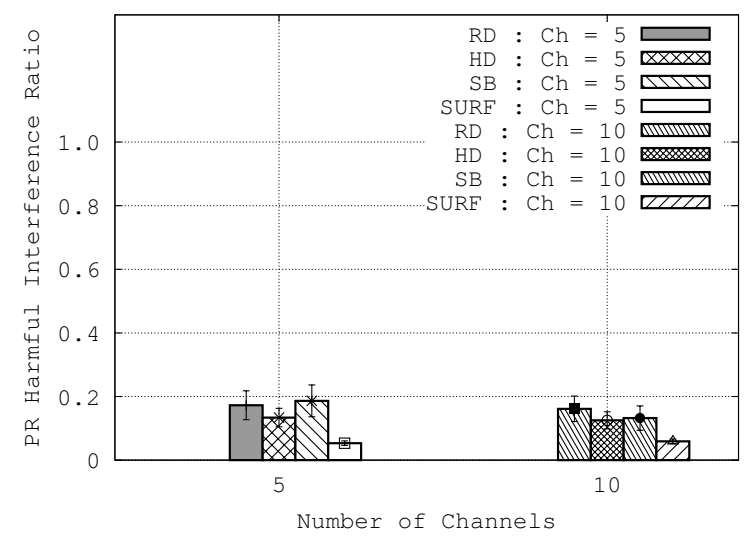

(a)

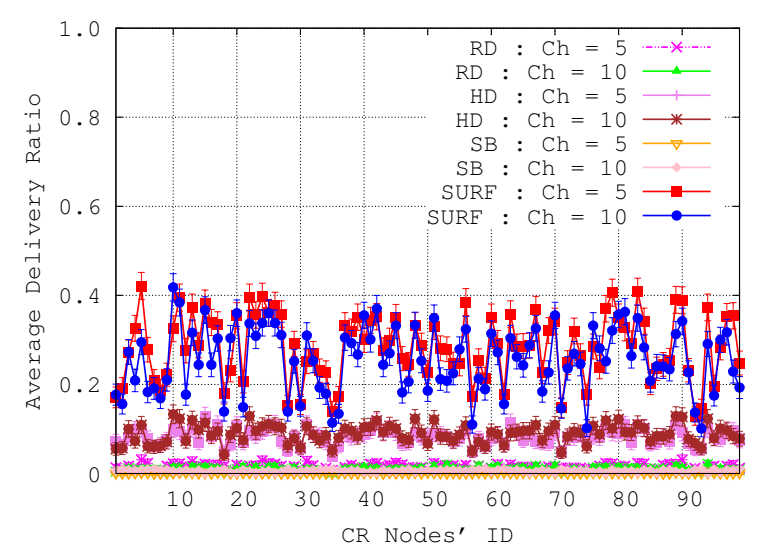

(b)

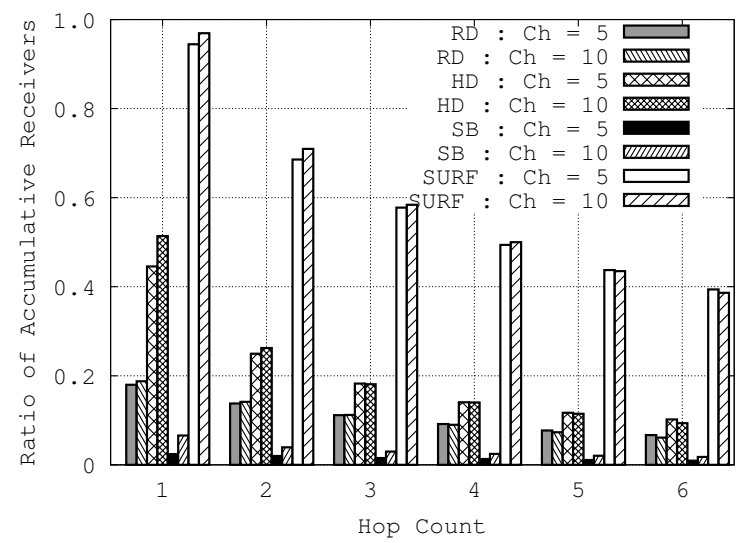

(c)

Figure 19: Low Primary Radio Activity. (a) PR harmful interference ratio for RD, HD, SB and SURF. (b) CR Nodes' ID and average delivery ratio for RD, HD, SB and SURF. (c) Hop count and Ratio of accumulative receivers for RD, HD, SB and SURF. 


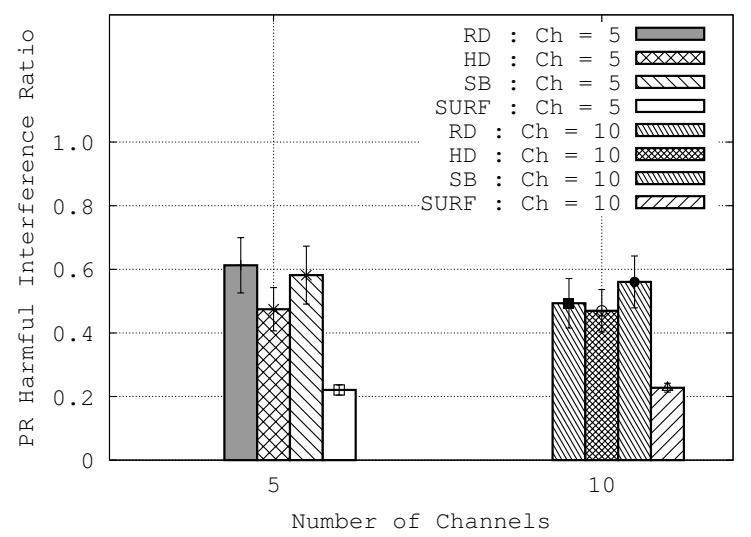

(a)

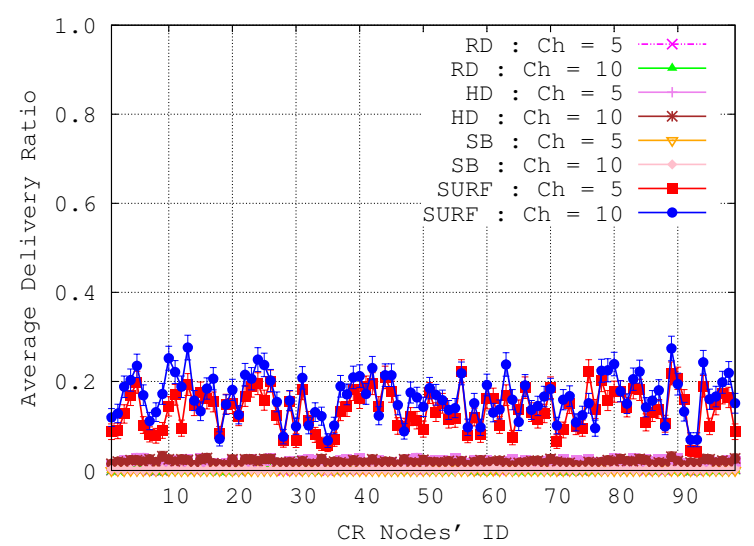

(b)

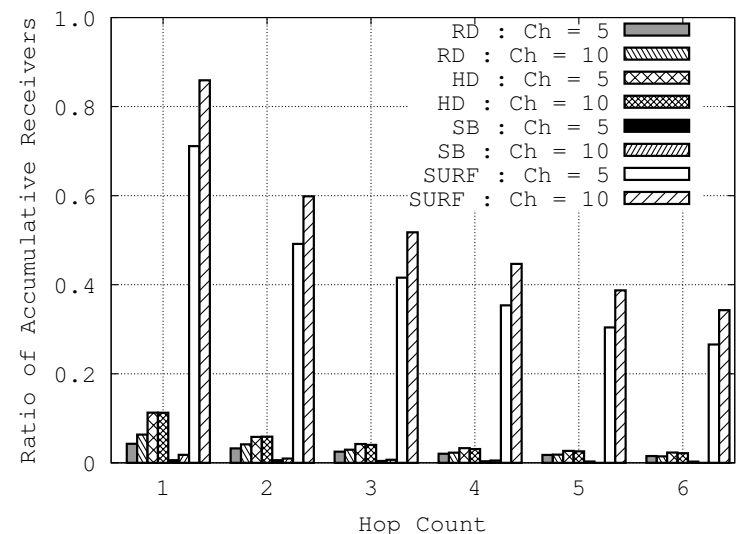

(c)

Figure 20: Intermittent Primary Radio Activity. (a) PR harmful interference ratio for RD, HD, SB and SURF. (b) CR Nodes' ID and average delivery ratio for RD, HD, SB and SURF. (c) Hop count and Ratio of accumulative receivers for RD, HD, SB and SURF.

RR $n^{\circ} 7628$ 
Table 6: Harmful Interference Ratio (HIR) (in \%) under various Primary Radio Nodes Activity.

\begin{tabular}{c|cc|cc|cc|cc}
\hline & \multicolumn{2}{|c|}{$R D$} & \multicolumn{2}{c|}{$H D$} & \multicolumn{2}{c}{$S B$} & \multicolumn{2}{c}{ SURF } \\
\hline & $\mathbf{C h = 5}$ & $\mathbf{C h}=\mathbf{1 0}$ & $\mathbf{C h = 5}$ & $\mathbf{C h}=\mathbf{1 0}$ & $\mathbf{C h}=\mathbf{5}$ & $\mathbf{C h}=\mathbf{1 0}$ & $\mathbf{C h = 5}$ & $\mathbf{C h = 1 0}$ \\
\hline Long Term & 63 & 53 & 51 & 49 & 50 & 50 & 23 & 27 \\
\hline High & 90 & 87 & 86 & 83 & 89 & 89 & 60 & 65 \\
\hline Low & 17 & 16 & 13 & 12 & 18 & 13 & 5 & 5 \\
\hline Intermittent & 61 & 49 & 47 & 46 & 58 & 56 & 22 & 22 \\
\hline
\end{tabular}

\subsection{Performance Analysis}

This section presents the performance analysis of the fours channel selection strategies under varying PR nodes activity. To achieve this, we performed extensive NS-2 simulations. For this end, three performance metrics are considered:

1. Harmful Interference Ratio (HIR): This metric is defined in order to capture the notion of collision with PR nodes. HIR is defined as the ratio of the total number of times the channel is occupied by PR node after the channel selection decision over total number of times the channel selection decision occurs.

2. Average Delivery Ratio: This metric is defined to effectively measure the data dissemination process. It is the ratio of packets received by a particular CR node over total packets sent in the network.

3. Ratio of Accumulative CR Receivers: This metric also evaluates the data dissemination process. It is defined as the average ratio of accumulative $\mathrm{CR}$ receivers per hop over the accumulative effective neighbors per hop. Accumulative CR receivers per hop are the number of CR receivers per hop that successfully received the message, while accumulative effective neighbors per hop are the CR neighbors that selects the same channel for overhearing as the sender node used for transmission. Note that by accumulative ratio we mean: at each new hop $h$, the receivers and effective neighbors of all previous hops $l<h$ are summed up to the ones at hop $h$.

The number of $\mathrm{CR}$ nodes is fixed to $\mathrm{N}=100$. CRs are randomly deployed within a square area of $a^{2}=700 \times 700 \mathrm{~m}^{2}$ and their transmission range is set to $R=250 \mathrm{~m}$. Simulations run for 1000 seconds and a total of 1000 packets are sent, where each packet is sent by a randomly selected node at an interval of 1 second. All results are obtained with a confidence interval of $95 \%$.

We consider $5(C h=5)$ and $10(C h=10)$ total number of channels, which allows varying the neighborhood density $d_{\text {avg }}$ between 11.3 (when $\mathrm{Ch}=5$ ) and 20.1 (when $\mathrm{Ch}=10$ ). Note this density is computed after the spectrum sensing provides the list of available channels and before the CRs select the channel to transmit/overhear. In this case, it is worth mentioning that, at the following simulation studies, the neighborhood 
density varies in function of the CRs' channel selection and is lower than the above ones. The results attest the obtained low delivery ratios are mainly due to the creation of different topologies resulted from the multi-channel availability and distributed channel selection by CRs. This can be verified in the Fig. 16, which shows results for delivery ratio, number of receivers and of effective neighbors, for $\mathrm{Ch}=5$ and $\mathrm{Ch}=10$ when no $P R$ nodes activity is present in the channels. As can be observed, even when CR nodes do not have to compete with PR nodes to have access to the channels, the average delivery ratio ranges from $35 \%-50 \%$, the average number of effective neighbors ranges from $10-20$ and the average number of receivers ranges from $12-2$ (from $1^{\text {st }}$ to $6^{\text {th }}$ hop) in SURF.

Fig. 17-Fig. 20 show the graphs for varying PR nodes activity patterns. Similarly, Table 6 summarizes the harmful interference ratio of Fig. 17-Fig. 20. In Long Term PR activity, besides of guaranteeing lower HIR compared to RD, HD, and SB, SURF also ensures a higher delivery ratio than such approaches. In High PR activity, all the channel are highly occupied, and consequently, very less chances for communication is let to all the approaches. Nevertheless, SURF is able to manage very low HIR and still have some delivery ratio ( $2 \%$ around), compared to the other approaches.

It is clear that when PR activity is very low (cf Fig. 19) every strategy behaves well in term of HIR (cf. 19(a)). In this case, SURF helps select the best channel in term of CR connectivity, i.e., delivery ratio to CR (cf. Fig. 19(b)), while generates very less or almost zero HIR, when compared to $\mathrm{RD}, \mathrm{SB}$, and $\mathrm{HD}$. The receivers ratio is also the highest for SURF.

Unsurprisingly, the best performance gain is observed in the intermittent case when using SURF: Lower HIR and higher delivery ratio is provided than RD, HD, and SB. It is worth noting that, in the cases where short ON for PR nodes is considered (i.e., in intermittent or low activity scenarios), all the approaches perform the better. However, the channel selection mechanism provided by SURF could find the best spectrum opportunities in all considered cases, while respecting the PR nodes activities.

Main Conclusions. Conclusions are quite typical and are for every wireless system in general:

- When the system is free (Low PR activity), every solution offers acceptable gain. Sometimes a clever solution does not worth it due to the complexity it introduces.

- When the system is close to maximum capacity (High PR activity), all solutions have bad performance. When channels are fully occupied by PRs there is no real opportunity for transmission, here also the gain is very low compared to the complexity of the solutions.

- Intermittent case is the case where clever solutions need to operate. This is where SURF gives the best results and the target region to avail communication opportunities.

\subsection{Improvements regarding SURF}

The channel selection strategy provided by SURF can be further enhanced by considering the primary radio nodes activity pattern. In the previous section, we have pointed out that the intermittent case is the case where clever solutions need to operate. In this regard, we can evaluate the "power" of using other history-based metrics (that try to better infer the quality of channels) combined with the current $P_{w}$ of SURF. SURF is 
then required to keep track of history of past PR nodes activity. This history could be used to give more weight to the channels with short $\mathrm{ON}$ in average. Some examples of metrics are given below:

1) How often the channel is free? Here, SURF may keep history of channel states. SURF then considers an "observation time window". In this manner, SURF will compute the ratio of being free over the window (the size of the time window could be varied to evaluate the impact).

2) How long channels stay in OFF state? Here, SURF may compute the duration of OFF state over the total time in the considered time window. This metric depends on how SURF keeps the history of channel states. This could be done on single time slot basis or varying slots of time. This metric also depends upon when the verification for a free channel is performed (periodically or only when a packet event (reception or transmission) happens.

3) What was the ratio of success (reception or transmission) over the times the channel was in OFF state? This metric will give the quality of the channel in terms of contention, variability of PR activities, etc.

As plan of our future work, we intend to improve SURF by keeping the history of PR nodes activity. Moreover, we also plan to broaden our investigation on the impact of PR nodes activity on channel selection strategies. We want to achieve this by considering other PR activity models, such as Bernoulli Process, Beta Distribution etc. Real time PR nodes activity traces can also be included in our studies to broaden the scope of our investigation.

\section{Related Work}

Recently, a lot of channel selection strategies have been proposed for cognitive radio networks $[5,6,7,8,9,10,12,13,14,15,16]$. These channel selection strategies are designed to achieve different performance goals, for instance, optimization of throughput, delay, etc. Besides achieving these goals, each channel selection strategy has a nature, according to its reaction with the appearance of PR nodes on the CR communicating channel. Therefore, channel selection strategies can be classified into three categories by nature: (1) proactive (predictive), (2) threshold based, and (3) reactive. From the communication perspective, channel selection strategies can be classified into centralized and distributed. The classification of channel selection strategies in cognitive radio networks is shown in Fig. 21. Table 7 compares different channel selection strategies for cognitive radio networks and their features. In the following, we discuss each classification in detail.

\subsection{Goals of Channel Selection Strategies}

From the performance perspective, authors in [5] proposed a channel selection strategy to satisfy the traffic demands of Access Points. Several channel selection strategies have proposed in the literature for throughput maximization [16,47, 14, 8, 48]. In [14], the authors determined the transmission schedule of the CR nodes in order to improve the network throughput. In [16], the authors improved the throughput of the CR users in the TV broadcast network. In fact, the authors proposed a predictive channel selection scheme to maximize spectrum utilization and minimize disruptions to PR nodes. They considered a single-hop network in which CR nodes coordinates with the TV 
receiver to collect information regarding PR activity. Two opportunistic channel selection schemes, CSS-MCRA and CSS-MHRA, are proposed in [47]. In CSS-MCRA, the goal was to maximize the throughput while minimize the collision rate. In CSSMHRA, the goal was to maximize the throughput while minimize the handoff rate. CSS-MCRA and CSS-MHRA both considered single user and are predictive in nature.

Load balancing is another important goal of channel selection strategies $[11,49]$. In [11], the authors proposed a channel and power allocation scheme for CR networks. The objective was to maximize the sum data rate of all CRs. They considered the availability of a centralized authority, which monitors the PR activity and assign channels to CR nodes. Sensing-based and probability-based spectrum decision schemes are proposed in [49] to distribute the load of CR nodes to multiple channels. The authors derived the optimal number of candidate channels for sensing-based scheme and the optimal channel selection probability for probability-based spectrum decision scheme. The objective of both schemes was to minimize the overall system time of the CR users.

The authors in [35] proposed a predictive channel selection scheme to minimize the channel switching delay of a single CR node. Other channel selection strategies focus on optimizing the expected waiting time [50,51], remaining idle time [52,53], reduce system overhead and improve CR QoS [54]. A predictive channel selection strategy, Voluntary Spectrum Handoff (VSH) [52], is proposed to reduce the communication disruption duration due to handoffs and to select the channel that has maximum remaining idle time. However, VSH requires the presence of Spectrum Server (SS), a centralized entity, to monitor the activities of PR and CR nodes. In [12], the authors proposed a channel selection scheme to maximize the total channel utilization. In their paper, the authors consider source-destination pairs in single-hop context. Channel selection strategies can also be used in conjunction with routing protocols for reliable path selection [4] and good route selection for delay sensitive applications [55]. Both the channel selection schemes $[4,55]$ are designed to work with routing protocols, while in SURF, we consider channel selection scheme for broadcasting.

\subsection{Nature of Channel Selection Strategies}

In proactive channel selection strategies [7, 56, 34, 57, 58, 16, 35], the activity of PR nodes is predicted and the CR nodes move to the channel according to the prediction. In $[7,57]$, the authors classified the PR traffic and applied different prediction rules. These prediction rules were then used in the predictive channel selection scheme to find the channels with the longest idle times for CR use. In [58], the authors explored two approaches of predictive dynamic spectrum access (PDSA). Their basic goal was to predict when the channels will be idle, based on observations of the primary radio nodes channel usage. They determined the expected channel idle times for CR usage. Two proactive channel selection strategies, PRO-I and PRO-II are proposed in [34]. The goal of these schemes were to minimize disruptions to PRs and throughput maximization of CR nodes. The authors uses a single pair of CR nodes and they ignored the impact of other CR nodes contending for the channel. Note that in SURF, CR nodes select the channel which has highest probability of being in idle state.

The authors in [56] proposed a channel selection scheme that optimizes the delay in finding the channels using the history. Their scheme is based on two steps: the database step and the signal detection step. In the database step, the database collects information about the channels. The CR node, when required a channel for transmission, sends a query to the database. The database then provides the most probable 
unoccupied channels, which are the best candidates for searching the channels. These channels are then submitted to the CR node. The CR node then performs the power level detection, and when required, the full signal detection. CR node then selects the channels based on the priority.

Threshold based schemes are those channel selection schemes in which the PR nodes active all the time and no idle channel is available to CR nodes. In these schemes, $\mathrm{CR}$ nodes are allowed to share the channel as long as the interference caused by the CR nodes to the PR nodes is below a certain threshold. For instance, [14] is a threshold based channel selection scheme. In this scheme, the authors considered the TV broadcast network as a primary network. Each TV receiver is equipped with a sensor, which is responsible for monitoring the activity of TV receiver. This sensor then communicates the PR activity information to the CR nodes. CR nodes use this historical PR activity information to select the channel.

In reactive channel selection strategies [59, 60, 61, 62, 63], channel switching occurs after the PR node appears. In fact, in reactive channel selection schemes, CR nodes monitor local spectrum through individual or collaborative sensing $[61,62,63$, $64,65,66]$. After detecting a change in the spectrum, e.g., channel is occupied by PR node, CR node stop the transmission, return back the channel to the PR node and search for other channel to resume the transmission. In [67], the authors compared two types of spectrum handoff schemes: proactive and reactive spectrum handoff schemes. In reactive-sensing handoff scheme, the target channel is selected after the spectrum handoff request is made. While in proactive spectrum handoff scheme, the target channel is predetermined. The authors mentioned that the advantage of reactive spectrum handoff scheme resides in the accuracy of the selected target channel, but incurs the cost of sensing time. On the contrary, the proactive spectrum handoff scheme avoid the sensing time, but the pre-determined channel may not be available. In [68], the co-authors of [67] provided the modeling and analysis of reactive spectrum handoff scheme in more detail.

In [59], the authors proposed a sensing-based opportunistic channel access scheme. They considered a Primary TV broadcast network. They also considered a single PR node and a single CR node and a base station is required for keeping the primary channel's statistics. A reactive multi-channel mac protocol, RMC-MAC, for opportunistic spectrum access is proposed in [60]. Their objective was to increase the bandwidth utilization and to reduce the forced termination probability. However, they considered a single-hop CR network. Dynamic frequency hopping communities (DFHC) [69] is also a reactive approach, which is designed for IEEE 802.22 networks. DFHC is a single hop approach and requires the presence of base station.

\subsection{Channel Selection Strategies from the Communication Perspec- tive}

From the communication perspective, channel selection strategies can be classified into centralized and distributed. In [71], a comparison between centralized and distributed approaches for spectrum management is done. In centralized channel selection strategies, a centralized entity is present, which helps CR nodes in their channel selection decision, e.g., [72, 73, 74]. The authors in [75] investigated different steps for the development of centralized algorithms for different radio networks. In [5], the authors proposed an efficient spectrum allocation architecture that adapts to dynamic traffic 




Figure 21: Classification of channel selection strategies for Cognitive Radio Networks. 
Table 7: Channel selection strategies and their features.

\begin{tabular}{|c|c|c|c|}
\hline Strategy & Goal & Nature & Hop/User \\
\hline VSH [52] & Remaining idle time & Predictive & Centralized \\
\hline [16] & $\begin{array}{l}\text { Maximize channel utilization, throughput } \\
\text { maximization and minimize disruptions to PRs }\end{array}$ & Predictive & Single-hop \\
\hline SWIFT [10] & $\begin{array}{l}\text { Combine multiple non-contiguous unoccupied } \\
\text { bands to create a high-throughput wideband link }\end{array}$ & work on unlicensed band & N/A \\
\hline $\mathrm{CBH}, \mathrm{LH}[13]$ & $\begin{array}{l}\text { Maximize channel utilization \& decrease message } \\
\text { overhead }\end{array}$ & Reactive & Multi-hop \\
\hline WAIT [15] & Maximize throughput & Reactive & Single-hop \\
\hline CSS-MCRA [47] & $\begin{array}{l}\text { Minimize collision rate and Throughput } \\
\text { maximization }\end{array}$ & Predictive & Single user \\
\hline CSS-MHRA [47] & $\begin{array}{l}\text { Minimize handoff rate and Throughput } \\
\text { maximization }\end{array}$ & Predictive & Single user \\
\hline [14] & Throughput maximization & Threshold based & Centralized \\
\hline PS-OSA [48] & Throughput maximization & N/A & CR pairs \\
\hline [11] & Load balancing & Reactive & Centralized \\
\hline [49] & Load balancing & Predictive/Reactive & Single-hop \\
\hline [35] & Reduce channel switching delay & Predictive & Single user \\
\hline SCA-MAC [50] & Expected waiting time & Predictive & N/A \\
\hline POSH [51] & Expected waiting time & Predictive & N/A \\
\hline FLEX [5] & Traffic demands of Access Points & N/A & Single-hop \\
\hline IEEE $802.22[6]$ & $\begin{array}{c}\text { International wireless standard based on CR } \\
\text { technology to use TV spectrum without causing } \\
\text { harmful interference to TV devices }\end{array}$ & N/A & Centralized \\
\hline [53] & Remaining idle time & Proactive & CR pairs \\
\hline [54] & Reduce system overhead and improve CR QoS & N/A & N/A \\
\hline [12] & Maximize total channel utilization & Reactive & CR pairs \\
\hline MPP [4] & Reliable path selection & N/A & Multi-hop \\
\hline [55] & Route selection for delay sensitive applications & Reactive & Distributed \\
\hline [70] & Route selection for delay sensitive applications & Reactive & Distributed \\
\hline [7] & Find longest idle time channel & Predictive & N/A \\
\hline [56] & Optimize delay in finding the channel & Proactive & N/A \\
\hline PRO-I, PRO-II [34] & $\begin{array}{l}\text { Minimize disruptions to PRs, throughput } \\
\text { maximization }\end{array}$ & Proactive & Single pair \\
\hline [57] & $\begin{array}{c}\text { Reduce delay \& channel switching, maximize } \\
\text { throughput }\end{array}$ & Predictive & N/A \\
\hline PDSA [58] & To determine expected channel idle time & Predictive & N/A \\
\hline [59] & Outage requirement of $\mathrm{PR}$ user $\mathrm{CR}$ & Reactive & Centralized \\
\hline RMC-MAC [60] & $\begin{array}{c}\text { Reduce forced termination probability and } \\
\text { increase bandwidth utilization }\end{array}$ & Reactive & Single-hop \\
\hline DFHC [69] & Better QoS and maximize throughput & Reactive & Centralized \\
\hline SB [17] & Data Reachability & N/A & Distributed \\
\hline SURF & Data Reachability, minimize disruptions to PRs & Predictive & Distributed \\
\hline
\end{tabular}


demands but they considered a single-hop scenario of Access Points (APs) in Wi-Fi networks. An approach that use non-continuous unoccupied band to create a high throughput link is discussed in [10]. In [14], the authors proposed a centralized algorithm of channel sharing between CR nodes. Their algorithm is designed for sourcedestination pairs and is specially designed for single-hop communication. In their paper, the authors assumed that all the PRs are active all the time and no idle channel is available to $\mathrm{CR}$ nodes for their communication. A channel allocation scheme for IEEE 802.22 standard is proposed in [76]. However, this scheme is centralized in nature. The authors in [77] proposed an opportunistic channel selection scheme for IEEE 802.11based wireless mesh networks. However, an Access Point (AP) is required to connect the nodes to the Internet via mesh router.

In multi-hop cognitive radio networks, there is no centralized entity that helps CR nodes in their channel selection decision. Therefore, distributed channel selection strategies are required. Moreover, CR nodes have to rely on their locally inferred information and no cooperation or feedback is expected from the primary radio nodes. Due to PR activity, the holding time and the granularity of wireless spectrum bands also affects on multi-hop CR communications [22]. Thus, an adaptive channel selection strategy is required at both the sender and receiver node, so that the receiver node tuned to the right channel to receive sent information. All these factors makes channel selection in these networks extremely challenging, having very few works been done so far $[17,70,55]$. In $[70,55]$, the authors proposed a dynamic resource management scheme for multi-hop cognitive radio networks. In fact, their approach is a route/channel selection for delay sensitive applications, such a multimedia streaming, while SURF is a channel selection scheme for data dissemination and not for routing.

In selective broadcasting (SB) [17], each cognitive node selects a minimum set of channels (ECS) covering all of its geographic neighbors to disseminate messages in multi-hop cognitive radio networks. There are however, several challenges in the practicality of SB. Indeed, from the communication perspective, simultaneous transmission over a ECS requires more than one transceiver, which means having bigger and more complex devices, as it is done for military applications [18]. On the contrary, using a single transceiver to transmit over minimum set of channels requires determining the correct channel to overhearing a transmission, increases delay, and brings frequent channel switching. Secondly, from the perspective of overhearing, either neighbor nodes need to simultaneously overhear over multiple channels or synchronization is required among neighbors, which incurs scheduling overhead. Compared to these aforementioned channel selection approaches, SURF is predictive in nature. The goal of SURF is to achieve higher data reachability and minimize disruptions to PRs in the multi-hop network.

\section{Conclusion and Future Work}

In this paper, we have introduced SURF, an intelligent and distributed channel selection strategy for reliable data dissemination in multi-hop cognitive radio ad-hoc networks. The main design objective of SURF is the protection of primary radio nodes against harmful interference by CR transmissions and the increase of dissemination reliability in cognitive radio ad-hoc network. These two goals were achieved by classifying the channels on the basis of primary radio unoccupancy and the number of cognitive radio neighbors using each channel. Simulation results in NS-2 confirmed that SURF, when 
compared to random-based, higher degree, and selective broadcasting strategies, is effective in selecting the best channels. Furthermore, we show that unlike other solutions, SURF performance is enhanced with the increase of the number of existing channels. This is due to its intelligent selection mechanism. We intend in future to consider the traffic and data rates of CR nodes in the channel's weight calculation formula, as well as time needed to disseminate messages in the network.

\section{References}

[1] J. Mitola, G. Q. Maguire, Cognitive radio: Making software radios more personal, IEEE Personal Communications 6, no. 4 (1999) 13-18.

[2] FCC, Spectrum policy task force (sptf), report of the spectrum efficiency wg, Nov. 2002.

[3] I. F. Akyildiz, W.-Y. Lee, M. C. Vuran, S. Mohanty, Next generation/dynamic spectrum access/cognitive radio wireless networks: a survey, Computer Networks: The International Journal of Computer and Telecommunications Networking 50 , Issue 13 (2006) 2127 - 2159.

[4] H. Khalife, S. Ahuja, N. Malouch, M. Krunz, Probabilistic path selection in opportunistic cognitive radio networks, in: proceedings of the IEEE globecom conference, 2008, pp. 1-5.

[5] L. Yang, L. Cao, H. Zheng, E. Belding, Traffic-aware dynamic spectrum access, in: Proceedings of The Fourth International Wireless Internet Conference (WICON 2008), Hawaii, USA, 2008.

[6] C. Cordeiro, K. Challapali, D. Birru, S. N. Shanka, Ieee 802.22: An introduction to the first wireless standard based on cognitive radios, Journal of Communications 1 (1) (2006) 38-47.

[7] M. Hoyhtya, S. Pollin, A. Mammela, Classification-based predictive channel selection for cognitive radios, in: IEEE ICC, 2010.

[8] Q. Zhao, L. Tong, A. Swami, Y. Chen, Decentralized cognitive mac for opportunistic spectrum access in ad hoc networks: A pomdp framewrok, IEEE Journal on Selected Areas in Communications 25, No. 3 (2007) 589-600.

[9] D. Niyato, E. Hossain, Competitive spectrum sharing in cognitive radio networks: A dynamic game approach, IEEE Transactions on wireless communications 7 (7) (July 2008) 2651-2660.

[10] H. Rahul, N. Kushman, D. Katabi, C. Sodini, F. Edalat, Learning to share: Narrowband-friendly wideband wireless networks, in: ACM SIGCOMM, Vol. 38 , Issue 4, SEATTLE, WA, USA, 2008, pp. 147-158.

[11] H. Wang, J. Ren, T. Li, Resource allocation with load balancing for cogntive radio networks, in: IEEE GlobeCom, 2010.

[12] F. Hou, J. Huang, Dynamic channel selection in cognitive radio network with channel heterogeneity, in: IEEE Globecom, 2010. 
[13] V. S. Rao, R. V. Prasad, C. Yadati, I. Niemegeers, Distributed heuristics for allocating spectrum in cr ad hoc networks, in: IEEE Globecom, 2010.

[14] G. D. Nguyen, S. Kompella, Channel sharing in cognitive radio networks, in: MILCOM, 2010.

[15] P. Anggraeni, N. Mahmood, J. Berthod, N. Chaussonniere, L. My, H. Yomo, Dynamic channel selection for cognitive radios with heterogenous primary bands, Wireless Personal Communications 45 (2008) 369-384, 10.1007/s11277-0089464-7.

URL http: //dx.doi.org/10.1007/s11277-008-9464-7

[16] P. A. K. Acharya, S. Singh, H. Zheng, Reliable open spectrum communications through proactive spectrum access, in: TAPAS, 2006.

[17] Y. R. Kondareddy, P. Agrawal, Selective broadcasting in multi-hop cognitive radio networks, in: IEEE Sarnoff Symposium, Princeton, New Jersy, 2008, pp. 1-5.

[18] O. Younis, L. Kant, K. Chang, K. Young, Cognitive manet design for missioncritical networks, IEEE Communications Magazine (2009) 64-71.

[19] [link].

URL http://stuweb.ee.mtu.edu/ 1 jialian/

[20] [link].

URL http: //www. isi.edu/nsnam/ns/

[21] I. F. Akyildiz, W.-Y. Lee, K. R. Chowdhury, Crahns: Cognitive radio ad hoc networks, Ad Hoc Networks 7, issue 5 (2009) 810-836.

[22] H. Khalife, N. Malouch, S. Fdida, Multihop cognitive radio networks: to route or not to route, IEEE Networks (2009) 20-25.

[23] H. Harada, A small-size software defined cognitive radio prototype, in: Proceedings of the IEEE International Symposium on Personal, Indoor and Mobile Radio Communications (PIMRC), Cannes, France, 2008, pp. 1-5.

[24] K. G. Shin, H. Kim, C. Cordeiro, K. Challapali, An experimental approach to spectrum sensing in cognitive radio networks with off-the-shelf ieee 802.11 devices, in: 4th IEEE Consumer Communications and Networking Conference, CCNC 2007., 2007, pp. 1154 -1158. doi : 10.1109/CCNC. 2007.232.

[25] T. Yucek, H. Arslan, A survey of spectrum sensing algorithms for cognitive radio applications, IEEE Communications Surveys and Tutorials 11, Issue No. 1 (First Quarter 2009) 116-130.

[26] W.-Y. Lee, I. Akyildiz, Optimal spectrum sensing framework for cognitive radio networks, IEEE Transactions on Wireless Communications 7 (10) (2008) 3845 -3857. doi:10.1109/T-WC. 2008.070391.

[27] G. Yuan, R. Grammenos, Y. Yang, W. Wang, Performance analysis of selective opportunistic spectrum access with traffic prediction, IEEE Transactions on Vehicular Technology 59 (4) (2010) 1949 -1959. doi:10.1109/TVT .2009.2039155. 
[28] A. W. Min, K. G. Shin, Exploiting multi-channel diversity in spectrum-agile networks, in: Proceedings of INFOCOM, 2008, pp. 1921 - 1929.

[29] H. Kim, K. Shin, Efficient discovery of spectrum opportunities with mac-layer sensing in cognitive radio networks, IEEE Transactions on Mobile Computing 7 (5) (2008) 533 -545. doi : 10.1109/TMC. 2007.70751.

[30] S. L. Loukas Lazos, M. Krunz, Spectrum opportunity-based control channel assignment in cognitive radio networks, in: 6th Annual IEEE Communications Society Conference on Sensor, Mesh and Ad Hoc Communications and Networks (SECON 2009)., Rome, Italy, 2009.

[31] H. Kim, K. Shin, Fast discovery of spectrum opportunities in cognitive radio networks, in: 3rd IEEE Symposia on New Frontiers in Dynamic Spectrum Access Networks (IEEE DySPAN), October, 2008., pp. 1-12.

[32] O. Mehanna, A. Sultan, H. E. Gamal, Cognitive mac protocols for general primary network models, CoRR abs/0907.4031.

[33] A. S. Zahmati, X. Fernando, A. Grami, Steady-state markov chain analysis for heterogeneous cognitive radio networks, in: Proceedings of the 33rd IEEE conference on Sarnoff, Sarnoff'10, IEEE Press, Piscataway, NJ, USA, 2010, pp. 107-111.

URL http: //portal .acm.org/citation. cfm?id=1843486.1843507

[34] L. Yang, L. Cao, H. Zheng, Proactive channel access in dynamic spectrum networks, Elsevier Physical Communications Journal vol (1) (2008) 103-111.

[35] Q. D. Xue Feng, S. Guangxi, L. Yanchun, Smart channel swiching in cognitive radio networks, in: CISP, 2009.

[36] B. Vujicic, N. Cackov, S. Vujicic, L. Trajkovic, Modeling and characterization of traffic in public safety wireless networks, in: In Proc. of SPECTS, 2005, pp. 214-223.

[37] [link].

URL http://www.fcc.gov/pshs/public-safety-spectrum/700-MHz/

[38] S. Geirhofer, L. Tong, B. M. Sadler, Dynamic spectrum access in wlan channels: Emperical model and its stochastic analysis, in: ACM TAPAS, 2006.

[39] A. Adas, Traffic models in broadband networks, IEEE Communications Magazine 35 (7) (1997) 82 -89. doi:10.1109/35.601746.

[40] K. Sriram, W. Whitt, Characterizing superposition arrival processes in packet multiplexers for voice and data, IEEE Journal on Selected Areas in Communications 4 (6) (1986) 833-846. doi : 10.1109/JSAC. 1986.1146402.

[41] C. Arachchige, S. Venkatesan, N. Mittal, An asynchronous neighbor discovery algorithm for cognitive radio networks, in: New Frontiers in Dynamic Spectrum Access Networks, 2008. DySPAN 2008. 3rd IEEE Symposium on, 2008, pp. 1 -5. doi: 10.1109/DYSPAN. 2008.78. 
[42] N. Mittal, S. Krishnamurthya, R. Chandrasekarana, S. Venkatesana, Y. Zenga, On neighbor discovery in cognitive radio networks, Journal of Parallel and Distributed Computing 69, issue 7 (July 2009) 623-637.

[43] M. Di Felice, K. R. Chowdhury, L. Bononi, Modeling and performance evaluation of transmission control protocol over cognitive radio ad-hoc networks, in: MSWiM '09, ACM, New York, NY, USA, 2009, pp. 4-12. doi:http://doi.acm.org/10.1145/1641804.1641809. URL http://doi.acm.org/10.1145/1641804.1641809

[44] S. Bayhan, F. Alagöz, Distributed channel selection in crahns: A non-selfish scheme for mitigating spectrum frag Ad Hoc Networks In Press, Corrected Proof (2011) -. doi:DOI : 10.1016/j.adhoc.2011.04.010.

URL http://www.sciencedirect.com/science/article/pii/S1570870511000941

[45] J. Riihijarvi, J. Nasreddine, P. Mahonen, Impact of primary user activity patterns on spatial spectrum reuse opportunities, in: European Wireless Conference (EW 2010), 2010.

[46] M. H. Rehmani, A. C. Viana, H. Khalife, S. Fdida, Improving data dissemination in multi-hop cognitive radio ad-hoc networks, in: 3rd International ICST Conference on Ad Hoc Networks (ADHOCNETS 2011), Paris, France - (To appear)., 21-23 Sep 2011.

[47] Q. Xiao, Y. Li, M. Zhao, S. Zhou, J. Wang, Opportunistic channel selection approach under collision probability constraint in cognitive radio systems, Computer Communications 32 (18) (2009) 1914-1922.

[48] Q. Zhao, S. Geirhofer, L. Tong, B. M. Sadler, Opportunistic spectrum access via periodic channel sensing, IEEE Transactions on Signal Processing 56 (2) (2008) $785-796$.

[49] L.-C. Wang, C.-W. Wang, F. Adachi, Load-balancing spectrum decision for cognitive radio networks, IEEE Journal on Selected Areas in Communications 29 (4) (2011) 757-769.

[50] A. C.-C. Hsu, D. S.-L. Wei, C.-C. J. Kua, A cognitive mac protocol using statistical channel allocation for wireless ad-hoc networks, in: WCNC, 2007.

[51] R.-T. Ma, Y.-P. Hsu, K.-T. Feng, A pomdp-based spectrum handoff protocol for partially observable cognitive radio networks, in: WCNC, 2009.

[52] S.-U. Yoon, E. Ekici, Voluntary spectrum handoff: A novel approach to spectrum management in crns, in: ICC, 2010.

[53] Y. Song, J. Xie, Common hopping based proactive spectrum handoff in cogntive radio ad hoc networks, in: GlobeCom, 2010.

[54] P. Zhu, J. Li, X. Wang, A new channel parameter for cognitive radio, in: CrownCom, 2007.

[55] H. P. Shiang, M. V. D. Schaar, Delay-sensitive resource management in multi-hop cognitive radio networks, in: IEEE DySpan, 2008. 
[56] J. Vartiainen, M. Hoyhtya, J. Lehtomaki, T. Braysy, Priority channel selection based on detection history database, in: CROWNCOM, 2010.

[57] M. Hoyhtya, S. Pollin, A. Mammela, Performance improvement with predictive channel selection for cognitive radios, in: First International Workshop on Cognitive Radio and Advanced Spectrum Management,CogART 2008., Denmark, 2008, pp. 1-5.

[58] T. C. Clancy, B. D. Walker, Predictive dynamic spectrum access, in: Proc. SDR Forum Technical Conference, Orlando, Florida, USA, 2006.

[59] X. Liu, S. N., Sensing-based opportunistic channel access, Mobile Networks and Applications 11 (2006) 577-591, 10.1007/s11036-006-7323-x. URL http://dx .doi.org/10.1007/s11036-006-7323-x

[60] S. Fourati, S. Hamouda, S. Tabbane, Rmc-mac: A reactive multi-channel mac protocol for opportunistic spectrum access, in: 4th IFIP International Conference on New Technologies, Mobility and Security (NTMS), 2011, pp. $1-5$. doi:10.1109/NTMS.2011.5721056.

[61] S. M. Mishra, A. Sahai, R. Brodersen, Cooperative sensing among cognitive radios, in: IEEE ICC, 2006.

[62] N. H. A. Sahai, R. Tandra, Some fundamental limits on cognitive radios, in: 42 Allerton conference on Communication, Control and Computing, 2004.

[63] R. T. A. Sahai, N. Hoven, Opportunistic spectrum use for sensor networks: the need for local cooperation, in: IPSN, 2006.

[64] G. Ganesan, Y. G. Li, Cooperative spectrum sensing in cognitive radio networks, in: IEEE DySPAN, November 2005.

[65] A. Ghasemi, E. S. Sousa, Collaborative spectrum sensing for opportunistic access in fading environments, in: IEEE DySPAN, November 2005.

[66] D. Cabric, S. M. Mishra, R. W. Brodersen, Implementation issues in spectrum sensing for cognitive radios, in: Proceedings of Asilomar conference on signals, systems and computers, 2004.

[67] L.-C. Wang, C.-W. Wang, Spectrum handoff for cognitive radio networks: Reactive-sensing or proactive-sensins?, in: IEEE International Performance, Computing and Communications Conference (IPCCC 2008), 2008.

[68] C.-W. Wang, L.-C. Wang, F. Adachi, Modeling and analysis for reactivedecision spectrum handoff in cognitive radio networks, in: IEEE Global Telecommunications Conference (GLOBECOM 2010), 2010, pp. $1-6$. doi: 10.1109/GLOCOM. 2010.5683644.

[69] W. Hu, D. Willkomm, M. Abusubaih, J. Gross, G. Vlantis, M. Gerla, A. Wolisz, Cognitive radios for dynamic spectrum access - dynamic frequency hopping communities for efficient ieee 802.22 operation, IEEE Communications Magazine 45 (5) (2007) 80 -87. doi : 10 . 1109/MCOM . 2007. 358853. 
[70] J. Zhao, H. Zheng, G. H. Yang, Distributed coordination in dynamic spectrum allocation networks, in: First IEEE International Symposium on New Frontiers in Dynamic Spectrum Access Networks (DySPAN), Baltimore, Maryland, USA, 2005, pp. 259-268.

[71] G. Salami, A. Attar, a. R. T. Oliver Holland, H. Aghvami, A comparison between the centralized and distributed approaches for spectrum management, IEEE Communications Surveys and Tutorials 13, No. 2 (2011) 274-290.

[72] M. Pereirasamy, J. Luo, M. Dillinger, C. Hartmann, Dynamic inter-operator spectrum sharing for umts fdd with displaced cellular networks, in: IEEE Wireless Communications and Networking Conference, Vol. 3, 2005, pp. 1720 - 1725 Vol. 3. doi:10.1109/WCNC.2005.1424772.

[73] M. Buddhikot, K. Ryan, Spectrum management in coordinated dynamic spectrum access based cellular networks, in: First IEEE International Symposium on New Frontiers in Dynamic Spectrum Access Networks, DySPAN'05, 2005, pp. 299 -307. doi: 10.1109/DYSPAN . 2005.1542646.

[74] R. Kulkarni, S. A. Zekavat, Traffic aware inter vendor dynamic spectrum allocation: performance in multi-vendo in: Proceedings of the 2006 international conference IWCMC '06, ACM, New York, NY, USA, 2006, pp. 85-90. doi:http://doi.acm.org/10.1145/1143549.1143569. URL http://doi.acm.org/10.1145/1143549.1143569

[75] P. Leaves, K. Moessner, R. Tafazolli, D. Grandblaise, D. Bourse, R. Tonjes, M. Breveglieri, Dynamic spectrum allocation in composite reconfigurable wireless networks, IEEE Communications Magazine 42 (5) (2004) 72 - 81. doi:10.1109/MCOM. 2004.1299346.

[76] J. Zhu, S. Li, Channel allocation mechanisms for cognitive radio networks via repeated multi-bid auction, in: ICWMMN 2006, 2006.

[77] D. Niyato, E. Hossain, Cognitive radio for next-generation wireless networks: an approach to opportunistic channel selection in ieee 802.11based wireless mesh, IEEE Wireless Communications 16 (1) (2009) 46 -54. doi: 10.1109/MWC. 2009.4804368.

\section{Contents}

1 Introduction $\quad 3$

2 Challenges of Data Dissemination in Cognitive Radio Networks 5

3 System Model and Assumptions 6

3.1 Network Model . . . . . . . . . . . . . . . . . . . . . . 6

3.2 Spectrum Sensing by Cognitive Radio Nodes . . . . . . . . . . . . . 7

3.3 Primary Radio Activity or Wireless Channel Model . . . . . . . . . . 7

3.4 Exchange of Hello Packets . . . . . . . . . . . . . . . 7 
4 Channel Selection Strategy SURF 8

5 Primary Radio Unoccupancy 9

5.1 Recovery from Bad Channel Selection Decisions _... . . . . . . 10

6 Cognitive Radio Occupancy 12

7 Performance Analysis $\quad 13$

7.1 Implementation Setup . . . . . . . . . . . . . . . . . . . . 13

7.2 Performance Metrics . . . . . . . . . . . . . . . . . . . . . 14

7.3 Simulation Environment . . . . . . . . . . . . . . . . . 15

7.4 SURF Parameters Evaluation . . . . . . . . . . . . . . . . . . 16

7.4.1 Retries in SURF . . . . . . . . . . . . . . . . 17

7.4.2 Impact of Varying Neighborhood Density on SURF . . . . . . 17

7.4.3 PR Utilization of the Selected Channel . . . . . . . . . . . 18

7.5 SURF Comparison . . . . . . . . . . . . . . . . . . . 19

7.5.1 Protection to Primary Radio Nodes _ . . . . . . . . . . 19

7.5.2 Reliable Data Dissemination . . . . . . . . . . . . . . 21

7.5.3 Tuning of Sender and Receiver . . . . . . . . . . . . . . 22

7.5 .4 Packet Ratio . . . . . . . . . . . . . . . . . . . 23

8 Activity pattern impact of PR Nodes on Channel Selection Strategies 26

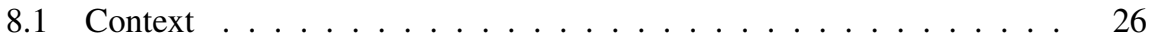

8.2 Channel Selection Strategies _ . . . . . . . . . . . . . . . 26

8.3 Primary Radio Nodes Activity Pattern _. . . . . . . . . . . . . 27

8.4 Performance Analysis . . . . . . . . . . . . . . . . . . . . . 34

8.5 Improvements regarding SURF . . . . . . . . . . . . . . 35

9 Related Work 36

9.1 Goals of Channel Selection Strategies _ . . . . . . . . . . . . 36

9.2 Nature of Channel Selection Strategies . . . . . . . . . . . . . . 37

9.3 Channel Selection Strategies from the Communication Perspective . . 38

10 Conclusion and Future Work 41 
Centre de recherche INRIA Saclay - Île-de-France

Parc Orsay Université - ZAC des Vignes

4, rue Jacques Monod - 91893 Orsay Cedex (France)

Centre de recherche INRIA Bordeaux - Sud Ouest : Domaine Universitaire - 351, cours de la Libération - 33405 Talence Cedex

Centre de recherche INRIA Grenoble - Rhône-Alpes : 655, avenue de l'Europe - 38334 Montbonnot Saint-Ismier

Centre de recherche INRIA Lille - Nord Europe : Parc Scientifique de la Haute Borne - 40, avenue Halley - 59650 Villeneuve d'Ascq

Centre de recherche INRIA Nancy - Grand Est : LORIA, Technopôle de Nancy-Brabois - Campus scientifique

615, rue du Jardin Botanique - BP 101 - 54602 Villers-lès-Nancy Cedex

Centre de recherche INRIA Paris - Rocquencourt : Domaine de Voluceau - Rocquencourt - BP 105 - 78153 Le Chesnay Cedex

Centre de recherche INRIA Rennes - Bretagne Atlantique : IRISA, Campus universitaire de Beaulieu - 35042 Rennes Cedex

Centre de recherche INRIA Sophia Antipolis - Méditerranée : 2004, route des Lucioles - BP 93 - 06902 Sophia Antipolis Cedex

Éditeur

INRIA - Domaine de Voluceau - Rocquencourt, BP 105 - 78153 Le Chesnay Cedex (France)

http://www.inria.fr

ISSN 0249-6399 